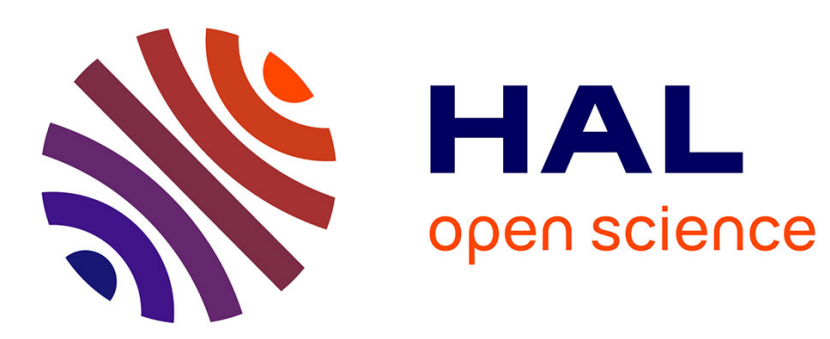

\title{
A continuum model for progressive damage in tough multinetwork elastomers
}

Shawn R Lavoie, Pierre Millereau, Costantino Creton, Rong Long, Tian Tang

\section{To cite this version:}

Shawn R Lavoie, Pierre Millereau, Costantino Creton, Rong Long, Tian Tang. A continuum model for progressive damage in tough multinetwork elastomers. Journal of the Mechanics and Physics of Solids, 2019, 125, pp.523-549. 10.1016/j.jmps.2019.01.001 . hal-02074568

\section{HAL Id: hal-02074568 https://hal.science/hal-02074568}

Submitted on 22 Mar 2019

HAL is a multi-disciplinary open access archive for the deposit and dissemination of scientific research documents, whether they are published or not. The documents may come from teaching and research institutions in France or abroad, or from public or private research centers.
L'archive ouverte pluridisciplinaire HAL, est destinée au dépôt et à la diffusion de documents scientifiques de niveau recherche, publiés ou non, émanant des établissements d'enseignement et de recherche français ou étrangers, des laboratoires publics ou privés. 


\title{
A continuum model for progressive damage in tough multinetwork elastomers
}

\author{
Shawn R. Lavoie†, Pierre Millereau§, Costantino Creton§, Rong Longł, Tian Tangł*,
}

†Department of Mechanical Engineering, 10-203 Donadeo Innovation Centre for Engineering, 9211-116 Street NW, Edmonton, AB, T6G 1H9, Canada

§ Laboratoire de Sciences et Ingénierie de la Matière Molle, CNRS, ESPCI Paris, PSL Research University, 10 rue Vauquelin, 75005 Paris, France

‡ Department of Mechanical Engineering, University of Colorado at Boulder, Boulder, CO, USA 80309.

*Corresponding author E-mail: tian.tang@ualberta.ca, Phone:780-492-5467, Fax: 780-492-2378

Keywords: Multinetwork elastomer, fracture toughness, progressive damage

\section{Abstract}

Recently a class of multinetwork elastomers (MNEs) was developed by swelling a filler polymer network with monomers that are subsequently polymerized to form matrix networks. Such MNEs were reported to possess remarkable stiffness and fracture toughness while maintaining the ability to sustain large deformation as found in simple elastomers. The enhancement in toughness is attained by prestretching the chains of the filler network through the introduction of one or more matrix network(s), thereby promoting energy dissipation through chain scission in the filler network. In this work, a model to capture the mechanical response of MNEs is developed, and validated with experimental data. Prestrech of the polymer chains is incorporated into the model by basing the strain energy density function on the combined effect of swelling and subsequent deformation of the completed MNE. The 
filler network is modeled as a polydisperse network of breakable polymer chains with nonlinear chain elasticity, while the matrix networks are modeled using the generalized neo-Hookean model. Although the filler network occupies only a small fraction of the material volume, the model shows that it contributes to the majority of the stress. Finally, the hysteresis during cyclic loading is shown to correlate with the accumulation of damage in the filler network during each cycle.

\section{Introduction}

Elastomers are widely utilized in many industrial and biomedical applications due to their ability to undergo large and reversible deformations. In most of these applications, from traditional industrial applications (e.g. tires) to emerging technologies (e.g. soft robotics and stretchable electronics), elastomeric components are required to sustain certain levels of mechanical loading. Therefore, it is desirable to design elastomers with high fracture toughness to enhance their load carrying capabilities. The first theory describing the intrinsic or threshold fracture of elastomers (in the absence of viscoelastic dissipation) was presented by Lake and Thomas [1], who stated that all bonds on a polymer strand between two crosslinks must be stretched to the breaking point before one bond ultimately fails. Thus, the energy to rupture a single polymer strand should scale linearly with the length of the strand $I$. As a result, the intrinsic fracture energy, estimated by multiplying the energy to rupture a single chain with the areal density of polymer chains across the fracture plane $\left(\sim l^{-1 / 2}\right)$, scales with the square root of the average chain length (i.e. $\sim l^{1 / 2}$ ). A consequence of this mechanism is that any attempt to increase the stiffness of an elastomer, by increasing the density of crosslinks and thereby decreasing the average chain length, will also make the elastomer more brittle with a decreased intrinsic toughness. This tradeoff between stiffness and toughness has been observed in many experimental data $[2,3]$. 
Another route to improve the fracture resistance of materials is to introduce bulk energy dissipation mechanisms, which can lead to the formation of a dissipation zone surrounding a crack [4]. The dissipation zone can prevent the energetic driving force for crack growth, supplied by external loading, from being fully delivered to the crack tip, which enhances the apparent fracture toughness without changing the intrinsic toughness. This is the underlying mechanism for toughness enhancement in filled elastomers $[5,6]$ where chains can attach and detach from filler particles embedded in the elastomer to dissipate energy, in viscoelastic elastomers [7] where molecular friction provides dissipation, and in interpenetrating networks where dissipation is introduced through the damage of sacrificial networks $[8,9]$. The last strategy, i.e. interpenetrating networks, has been implemented in numerous gel systems [8-12], but was only realized in elastomers very recently [13], where a variable fraction of prestretched chains can be built into the elastomer network to control the extent of chain rupture and energy dissipation.

Such interpenetrating multinetwork elastomers (MNEs), as described by Ducrot et al. [13], were created by first forming a crosslinked elastomer, i.e., the 'filler' network. This elastomer was then swollen using a solution containing monomers, during which chains of the filler network were stretched. These monomers were then polymerized in place to form a 'matrix' network interpenetrated with the filler network. This procedure was repeated to introduce additional matrix networks and further stretch the chains in the filler network. The terminology of matrix and filler networks is used by drawing an analogy to classical composites, where the filler material is of smaller fraction and is held together by the matrix to form the bulk material [14]. To monitor the extent of damage, bond rupture in the filler network around a crack tip was mapped by introducing light emitting photophores into the network. It was hypothesized that the prestretched filler network makes the dominant contribution to stress, while the matrix networks mainly serve to prevent large cracks from forming [13]. Validation of this 
hypothesis, however, is still difficult within the experimental instrumentation. Also, a systematic study of how physical parameters, such as degree of crosslinking and prestretch, impact macroscopic mechanical and fracture behaviors is necessary to optimize the stiffness and toughness of the MNEs, which is challenging with these types of time-consuming experiments. Hence, there is a need to develop quantitative models which can describe the mechanical response of this new class of materials. Such models will allow for an investigation of the relative contributions of each network to the stress. Implementation of such models in finite element analysis will allow for a systematic study of the impact of MNE parameters on the fracture toughness, e.g. how the size of the damage zone around crack tip depends on bulk material properties.

The objective of this work is to develop a nonlinear constitutive model capable of quantitatively predicting the mechanical behavior of MNEs, especially how the network pre-stretch induced by swelling affects the strain stiffening and damage in the MNEs. In particular, new experimental data are presented where three sets of double network and triple network elastomers are synthesized from a common parent single network elastomer. The pre-stretch of the first network is changed by varying the fraction of volatile solvent versus monomers during swelling, which enables a systematic study on the effect of pre-stretches. We will show that our model can capture all experimental data using a fixed set of model parameters not pertaining to swelling, thereby demonstrating predictive capability of our model.

Recently another model has been presented to capture the nonlinear mechanics of MNEs [15]. However, the model is phenomenological in nature and requires many fitting parameters to match the hysteresis measured in cyclic uniaxial tensile tests. Furthermore, in [15] the damage evolution was implemented by increasing the critical extension of the network which prevents the model from capturing the experimentally observed decrease in shear modulus due to chain rupture $[13,14]$. The key 
difference between our model and the previous one is that here the fundamental physics of polymer chains in the filler network is directly incorporated in the continuum model. Specifically, since the filler network contains a much higher density of crosslinks and experiences larger prestretch than the matrix networks, the chains in the filler network are expected to be highly stretched and subjected to stretch. Therefore, we implement a chain elasticity model that combines configurational entropy and bond deformation on the backbone of the polymer chain. Progressive damage in the MNEs is captured using a kinetic model describing chain rupture in the filler network. In contrast, the matrix networks contain relatively long and loosely crosslinked chains and thus are modeled using a generalized neo-Hookean model [16]. The prestretches experienced by the filler and matrix networks due to the swelling process are also taken into account. This physics based approach ensures that each parameter in our model has a physical interpretation, which can elucidate the connection between physical parameters (e.g., chain length) and continuum-level mechanical response.

The paper proceeds as follows. The experimental procedures to synthesize MNEs and the subsequent uniaxial tensile testing method are given in Section 2. Formulation of the model is presented in Section 3 which is divided to present the combined kinematics of swelling and MNE deformation in Section 3.1, and the material models in Section 3.2. In Section 4, the model is applied to uniaxial extension and is compared with experimental data. Conclusions are given in Section 5.

\section{Material and experimental methods}

\subsection{Reactants}

The monomer ethyl acrylate (EA) and the crosslinker 1,4-butanediol diacrylate (BDA) were purified over a column of activated alumina to remove the inhibitor. The UV initiator, 2-hydroxy-2- 
methylpropiophenone (HMP) was used as received. Ethyl acetate was used as the solvent. All reagents were purchased from Sigma Aldrich.

\subsection{Synthesis}

The synthesis of the MNEs was carried out in a glove box (Mbraun Unilab) under nitrogen atmosphere to avoid side reactions with oxygen in the air. Before introduction into the glove box, every reagent and solvent were bubbled with nitrogen for 45 minutes to remove the dissolved oxygen. The reaction was triggered by UV light (Vilbert Lourmat lamp, model VL-215.L, focuséd on $365 \mathrm{~nm}$ ). The UV power was kept low $\left(10 \mu \mathrm{W} / \mathrm{cm}^{2}\right)$ to create slow polymerization.

The preparation of MNEs is carried out in the following way starting from monomers, a first network (i.e. the filler network) is synthesised, and then multiple steps of swelling and polymerization are conducted to create a multiple network.

\subsubsection{Synthesis of the filler network}

The filler network was prepared from a solution in ethyl acetate consisting of: EA the monomer (50 wt \%), BDA the crosslinker (1.45 mol \% relative to monomer) and HMP the UV initiator (1 mol \% relative to monomer). The solution was cast in a $1 \mathrm{~mm}$ thick glass mold and the reaction was initiated by UV for 2 hours. After synthesis, the sample was washed and dried to remove unreacted species and free chains as described in $[13,17]$. This single network (SN) is then fully dried under vacuum at $80^{\circ} \mathrm{C}$.

\subsubsection{Preparation of MNEs with a controlled swelling of the filler network}


The synthesis method previously described in $[13,17]$ has been adapted to obtain a larger range of swelling states of the filler network. The filler network was swollen to equilibrium in a bath composed of monomer and solvent. At equilibrium, a swollen piece of the network was removed from the bath, sealed between PET sheets, and tightened between glass plates. Then a second polymerization, in a similar manner to that of the filler network, was conducted. Then the sample was dried under vacuum at $80^{\circ} \mathrm{C}$ overnight to remove the solvent. The resulting material is a double network (DN) elastomer, and its synthesis procedure is schematically depicted in Figure 1 . This procedure can be then repeated multiple times leading to the creation of a triple network (TN), a quadruple network (QN) and so on, with different volume fractions of each network. 
1: Synthesis of Filler Network

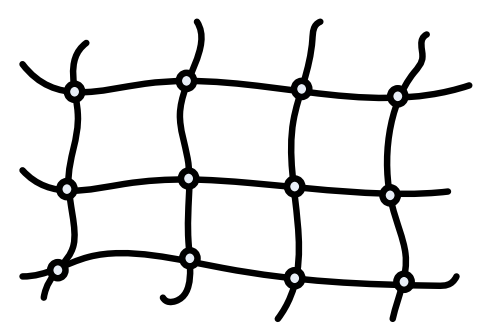

2: Swelling with Solvent and Monomers

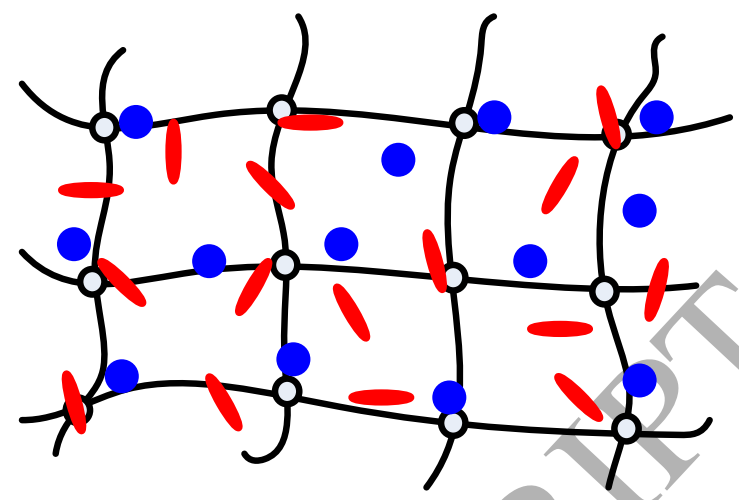

4: Drying of the Solvent

3: Polymerization at Equilibrium
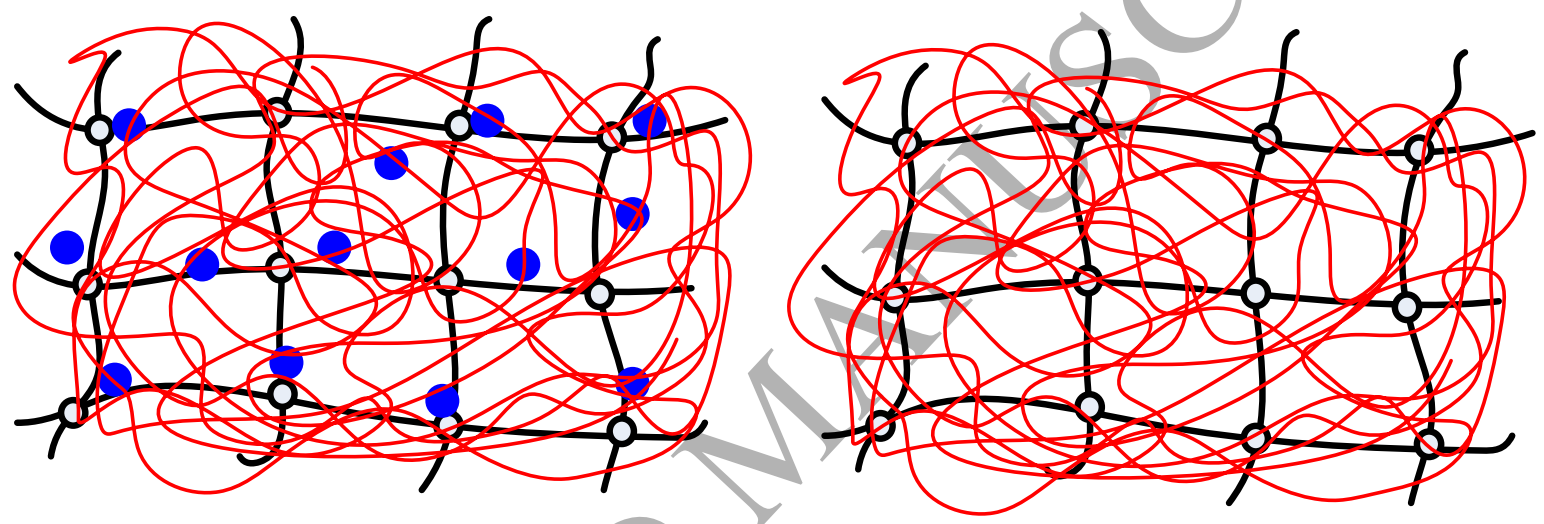

Figure 1: Schematics of the synthesis procedure of a double network elastomer

\subsection{Characterization of the synthesized materials}

To characterize the composition of synthesized MNEs, the mass and thickness of a sample are carefully measured after each step. Since in this work all the networks are comprised of the same 
monomer (ethyl acrylate), it is reasonable to assume that all networks have the same density (i.e., mass divided by the partial volume of the network). With the additional assumption of isotropic swelling, the prestretch of the filler network $\lambda_{0}$ can be quantified using the measured masses as follows

$$
\phi_{N}^{1}=\frac{m_{1}}{m_{N}}=\frac{1}{\lambda_{0}^{3}}
$$

where $\phi_{N}^{1}$ is the volume fraction of the filler network in an MNE consisting of $N$ networks, $m_{1}$ is the mass of the SN sample, and $m_{N}$ is the mass of the MNE sample. It should be noted that the model to be demonstrated in Section 3 is not limited to the assumption of same density in all networks, and is applicable to networks polymerized from different monomers. Two different ways of calculating the prestretches of the filler networks will be presented later, one being a generalization of Eq. (1) and the other based on the measurement of sample thickness.

\subsection{Mechanical Tests}

Mechanical tests were performed on a standard tensile Instron machine (model 5565) using a 100 $\mathrm{N}$ load cell. A video extensometer gave a local measurement of the stretch $\lambda=L / L_{0}$ where $L_{0}$ is the initial length and $L$ is the corresponding deformed length. The relative uncertainty of the measurements given by the load cell and the video extensometer are respectively $0.1 \%$ in the range of 0 to $100 \mathrm{~N}$ and $0.11 \%$ at the full scale of $120 \mathrm{~mm}$. Specimens were cut into a dumbbell shape using a normalized cutter (central part: length $20 \mathrm{~mm}$, cross-section $4 \mathrm{~mm}$ and thickness $0.6-2.5 \mathrm{~mm}$ depending on the sample). Uniaxial tensile tests from small to large strain were performed at a constant $500 \mu \mathrm{m} . \mathrm{s}^{-1}$ crosshead velocity and the typical stretch rate on the central part of the sample was around $\dot{\lambda}=0.04 \mathrm{~s}^{-1}$. 


\subsection{Set of materials}

Mechanical properties of the MNE can be influenced by several factors [13]: (i) number of networks, (ii) type of monomers used in each network, (iii) type of crosslinker, (iv) concentration of crosslinker, and (v) degree of swelling which can be changed by adding a certain amount of solvent to the solution of monomers. In this work, results from our theoretical modeling will be compared with experiments on MNEs in which all networks are comprised of Ethyl Acrylate (EA) but with different crosslinker concentrations and prestretches in the filler network. To be consistent with existing report [18], we use the following notation to denote different MNEs:

\section{$\operatorname{EAeX(Y)EA~}$}

EA: ethyl acrylate, monomer of the filler network

e: ethyl acetate has been used for the synthesis

$\mathbf{X}$ : mol \% of crosslinker used for the synthesis of filler network

$(\mathbf{Y}): \mathbf{Y}=\lambda_{0}$, prestretch of the filler network

EA: ethyl acetate, monomer of matrix networks.

A summary of the experimental datasets used in this work is given in Table 1. A tag is added to each elastomer for a simplified notation as well as to indicate the number of networks. We will be referring to these tags when comparing the modeling results with experiments later. From the base SN elastomer, three DN elastomers (DN1-3), with different pre-stretches, are created by controlling the ratio of 
monomer to solvent during swelling. Specifically, DN1, DN2 and DN3 were prepared by swelling the SN elastomer using mixtures with monomer volume fraction of $50 \%, 70 \%$ and $100 \%$, respectively. Three TN elastomers, with different pre-stretches, are synthesised by swelling the DN elastomers with pure monomers; e.g. TN1 is synthesised from DN1. Measurements were also taken for the matrix network alone (M), where the crosslinker concentration (0.01 mol\%) is 145 times less than that used in forming the filler network. Finally additional DN and TN data were extracted from Ducrot et al [13], DNP and TNP, where the synthesis procedure was similar to that of DN3 and TN3 with the exception that a photophore, chemiluminescent bis(adamantly)-1,2-dioxetane bisacrylate, was used ás crosslinker in place of BDA.

Table 1: Set of MNEs Investigated

\begin{tabular}{|c|c|c|c|c|c|}
\hline Sample name & $\lambda_{0}$ & $\begin{array}{l}\text { wt\% } \\
\text { of filler network }\end{array}$ & $\begin{array}{l}\text { Type of } \\
\text { network }\end{array}$ & $\begin{array}{c}\text { Number of } \\
\text { polymerization steps }\end{array}$ & Tag \\
\hline EAe1.45(1) & 1 & 10 & SN & 1 & SN \\
\hline EAe1.45(1.32)EA & & 42.0 & $\mathrm{DN}$ & 2 & DN1 \\
\hline EAe1.45(1.51)EA & 1.51 & 29.2 & $\mathrm{DN}$ & 2 & DN2 \\
\hline EAe1.45(1.68)EA & 1.68 & 20.5 & $\mathrm{DN}$ & 2 & DN3 \\
\hline EAe1.45(2.18)EA & 2.18 & 9.52 & $\mathrm{TN}$ & 3 & TN1 \\
\hline EAe1.45(2.41)EA & 2.41 & 7.39 & TN & 3 & TN2 \\
\hline EAe1.45(2.55)EA & 2.55 & 6.06 & $\mathrm{TN}$ & 3 & TN3 \\
\hline $\mathrm{EAe} 0.01(1)$ & 1 & 100 & Matrix & 1 & $\mathrm{M}$ \\
\hline EAe1.45(1.48)EA [13] & 1.48 & 30.8 & $\mathrm{DN}$ & 2 & DNP \\
\hline EAe1.45(2.72)EA [13] & 2.72 & 4.97 & TN & 3 & TNP \\
\hline
\end{tabular}




\section{Model}

\subsection{Kinematics}

\subsubsection{Swelling}

We number the networks, in superscripts, by the order $i(i=1 \ldots N)$ in which they were added to the material, where $N$ is the total number of networks which comprise the material: for a SN $N=1$, for $\operatorname{aDN} N=2$, and for a TN $N=3$. Each swelling and drying operation is denoted using the index $j$, in subscripts, which runs from 1 to a maximum value of $N-1$. The swelling and drying of a matrix network contains the following steps: (1) the networks is swollen to equilibrium by a solution containing monomers and solvent; (2) polymerization of the matrix network; and (3) the material is dried to evaporate the solvent. The relaxed configuration of each matrix network is the one after step (2) but before step (3) since drying introduces volume shrinkage of the material. The deformation gradient of each matrix network is measured relative to its relaxed configuration.

Figure 2a) shows the $j$ th swelling and drying operation and the associated deformation gradients: $\underline{\boldsymbol{\Phi}}_{j}^{\max }$ for the steps of swelling to equilibrium, $\underline{\boldsymbol{\Phi}}_{j}^{d r y}$ for drying and $\underline{\boldsymbol{\Phi}}_{j}=\underline{\boldsymbol{\Phi}}_{j}^{d r y} \underline{\boldsymbol{\Phi}}_{j}^{\max }$ for the entire procedure. Assuming that the swelling and drying are isotropic, the isotropic stretches during these steps $\left(\lambda_{j}^{\max }, \lambda_{j}^{d r y}\right.$ and $\left.\lambda_{s j}\right)$ can be calculated from

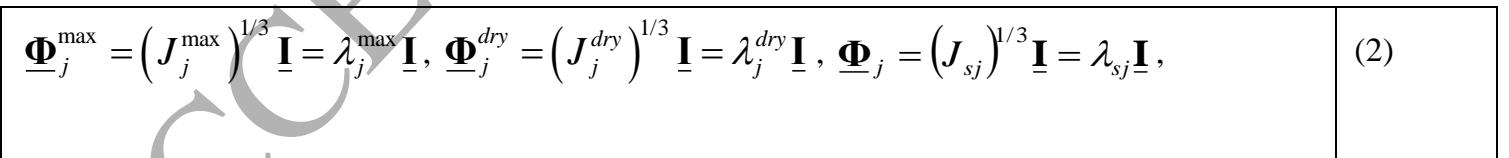

where $\underline{\mathbf{I}}$ is the second order identity tensor, $J_{j}^{\max }, J_{j}^{d r y}$ and $J_{s j}$ are respectively the volume ratios during the $j$ th swelling only, during the jth drying only, and during combined swelling and drying in the jth step. Clearly, $J_{s j}=J_{j}^{\max } J_{j}^{d r y}$ and $\lambda_{s j}=\lambda_{j}^{\max } \lambda_{j}^{d r y}$. Denote the volume fraction of monomers in the $j$ th swelling solution as $\xi_{j}^{m o n}$ then it follows that (see Supporting Information Section S1) 


$$
J_{j}^{d r y}=\frac{\xi_{j}^{m o n} J_{s j}}{J_{s j}-1+\xi_{j}^{m o n}}
$$

After the jth swelling and drying we obtain a MNE, and if the configuration at this point is set to be the reference configuration of the MNE, the deformation gradient that maps the ith network from its relaxed configuration to the reference configuration of the MNE is

$$
\underline{\boldsymbol{\Phi}}_{j}^{i}=\left[\prod_{k=i}^{j} \underline{\boldsymbol{\Phi}}_{k}\right] \underline{\boldsymbol{\Phi}}_{i-1}^{d r y}=\left(J_{i-1}^{d r y}\right)^{1 / 3}\left(\prod_{k=i}^{j}\left(J_{s k}\right)^{1 / 3}\right) \underline{\mathbf{I}}=\lambda_{i-1}^{d r y}\left(\prod_{k=i}^{j} \lambda_{s k}\right) \underline{\mathbf{I}}
$$

where the notation $\Pi$ indicates multiplication. For example, consider a TN elastomer just formed $(N=3$, $j=2$ ), the total swelling and drying deformation gradients are given by $\underline{\Phi}_{2}^{1}=\underline{\Phi}_{2} \underline{\Phi}_{1}$ for the filler network, $\underline{\boldsymbol{\Phi}}_{2}^{2}=\underline{\boldsymbol{\Phi}}_{2} \underline{\boldsymbol{\Phi}}_{1}^{d r y}$ for the second network, and $\underline{\boldsymbol{\Phi}}_{2}^{3}=\underline{\boldsymbol{\Phi}}_{2}^{d r y}$ for the third network. Note that the $\Pi$ notation returns 1 for an empty product. For example, for a SN elastomer $\underline{\boldsymbol{\Phi}}_{0}^{1}=\left(\prod_{k=1}^{0} \lambda_{s k}\right) \underline{\boldsymbol{\Phi}}_{0}^{d r y}=\underline{\mathbf{I}}$. Note that since the filler network was synthesized without using additional solvent, it is in the relaxed configuration after polymerization and thus $\underline{\boldsymbol{\Phi}}_{0}^{d r y}=\underline{\mathbf{I}}$. Using Eq. (4) we can define the ratio between the volume of the $i$ th network after the $j$ th swelling and drying and its volume when it is first introduced (before drying):

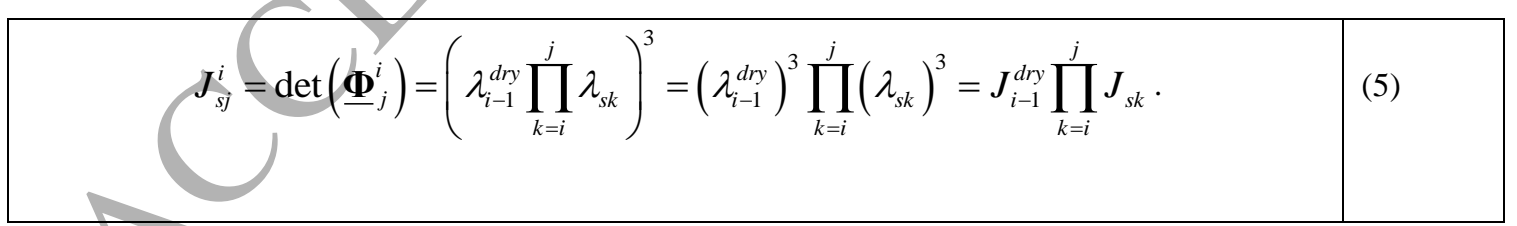

In writing the constitutive relationship for the MNE, the contribution from each network will be considered; hence it is necessary to determine the volume fraction of each network that makes up the total MNE volume. The volume fraction that the ith network occupies in the material when it is first introduced $(j=i-1)$ is given by 


$$
\begin{gathered}
\psi^{1}=1 \\
\psi^{i}=\xi_{i-1}^{m o n}\left(1-\left(J_{i-1}^{\max }\right)^{-1}\right) \quad i>1
\end{gathered}
$$

The first equation (for the filler network) is straightforward, while the second equation ( $i>1$ ) for the matrix networks can be explained as follows. After introduction of the ith network with the $(i-1)^{\text {th }}$ swelling, the volume of the MNE is increased by a factor of $J_{i-1}^{\max }$. Assuming all the original material remains intact after swelling, the volume fraction of the original material after this swelling becomes $\left(J_{i-1}^{\max }\right)^{-1}$. Since the newly introduced matrix network and solvent occupy the remaining volume $\left(1-\left(J_{i-1}^{\max }\right)^{-1}\right)$, the volume fraction of the matrix networks is given by $\xi_{i-1}^{\operatorname{mon}}\left(1-\left(J_{i-1}^{\max }\right)^{-1}\right)$ as stated by Eq. (6). Similarly, subsequent drying and introduction of additional matrix networks with the jth swelling causes the volume fraction of the existing networks to be divided by a factor of $J_{s j}$. Therefore, if we extend this idea the volume fraction of the ith network when $N$ networks are present $(j=N-1)$, denoted by $\phi_{N}^{i}$, is obtained by dividing the initial volume fraction $\psi^{i}$ by $J_{s(N-1)}^{i}$

\begin{tabular}{|l|l|l|}
\hline$\phi_{N}^{i}=\frac{\psi^{i}}{J_{s(N-1)}^{i}}$, & (7) \\
\hline
\end{tabular}
where $J_{s(N-1)}^{i}$ is given by Eq. (5). Note that Eq. (1) is a special case of Eq. (7).

\subsubsection{Deformation}

If the MNE, after all swelling operations $(j=N-1)$, is then mechanically deformed, the deformation gradient which maps the relaxed configuration of the $i^{\text {th }}$ network to the current configuration when $N$ networks are present is given by 


$$
\underline{\mathbf{F}}_{N}^{i}=\underline{\mathbf{F}}_{(N-1)}^{i}, \quad \text { (8) }
$$

where $\underline{\mathbf{F}}$ is the deformation gradient that maps the MNE configuration after all swelling and drying to its deformed configuration. Eq. (8) can be understood by considering the example of a TN elastomer for which a schematic of the deformation gradients is shown in Figure $2 b)$. Six configurations are shown: A) when the filler network is formed, $\mathrm{B}^{\prime}$ ) when the filler network has been swollen to equilibrium by a matrix network B) after drying to remove excess solvent from $B^{\prime}$ to form a $D N, C^{\prime}$ ) after the $D N$ material in configuration B has been swollen to equilibrium by an additional matrix network, C) after drying to remove excess solvent from $\mathrm{C}^{\prime}$ to form a TN, and $\mathrm{D}$ ) after the TN elastomer in configuration $\mathrm{C}$ has been mechanically deformed. A is the relaxed configuration for the filler network, $B^{\prime}$ is the relaxed configuration for the second network, and $\mathrm{C}^{\prime}$ is the relaxed configuration for the third network. $\mathrm{C}$ represents the reference configuration for the completed TN elastomer relative to which subsequent mechanical deformations are measured. 
a)

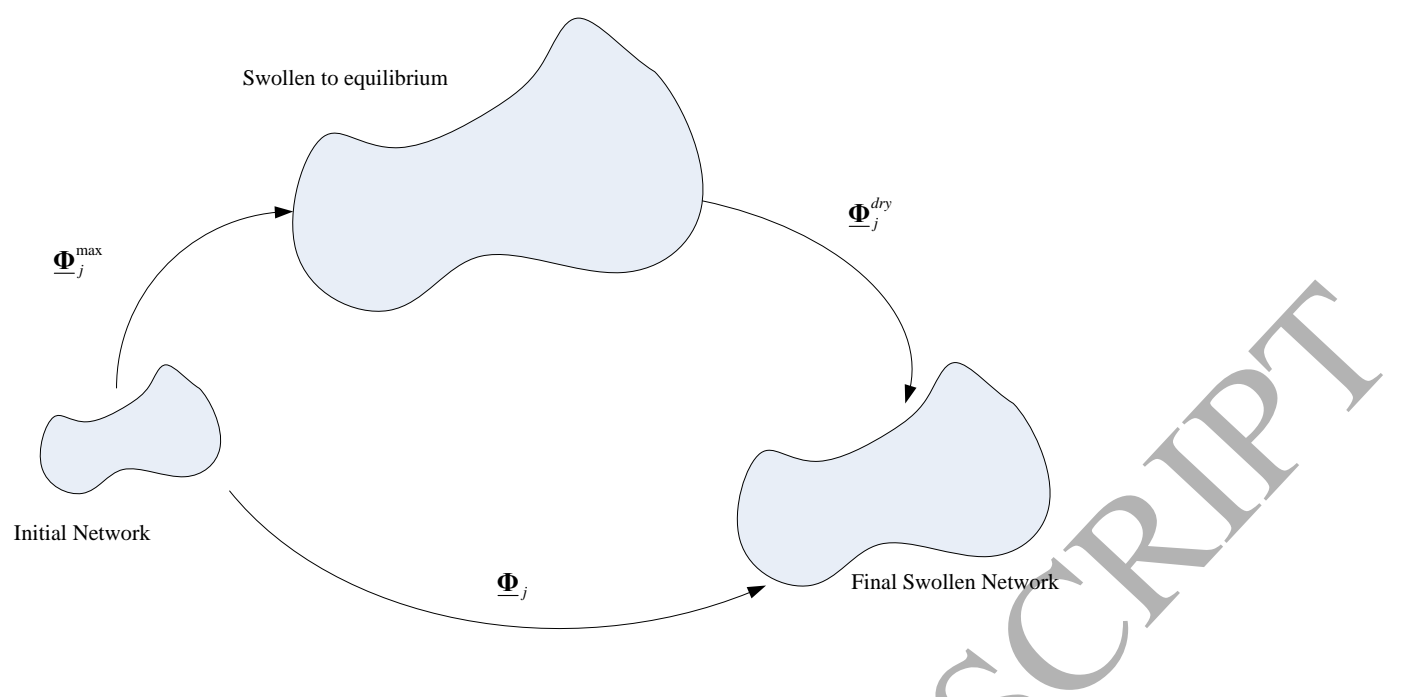

b)

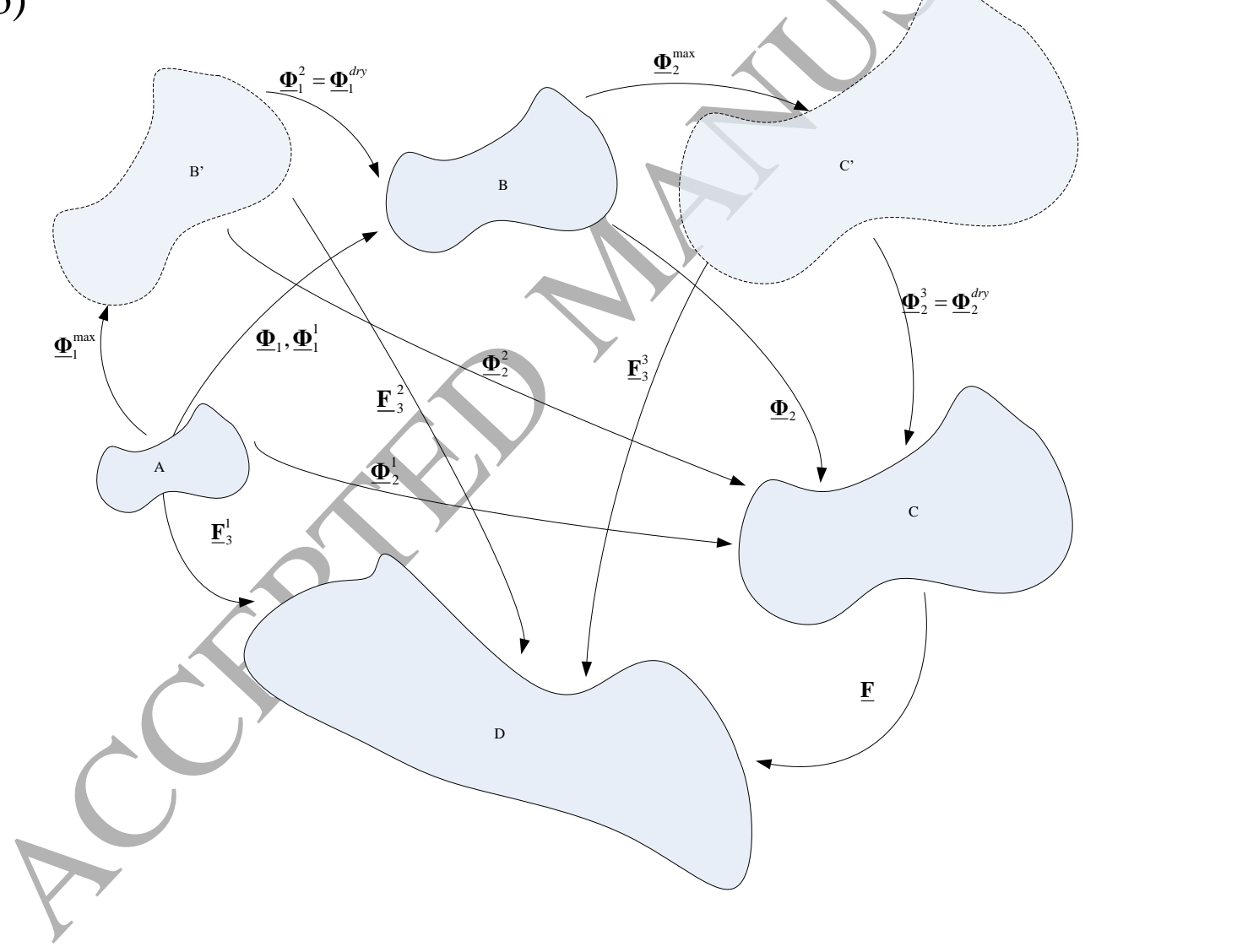

Figure 2: a) Swelling and drying in the jth step, and the corresponding deformation gradients. b) Deformation map for a TN elastomer showing relaxed configurations for the $1^{\text {st }}(A), 2^{\text {nd }}\left(B^{\prime}\right), 3^{\text {rd }}\left(C^{\prime}\right)$ networks and the deformed configuration $(D)$. The deformation gradients which relate these configuratiolns are shown schematically. 
Spatial forms of constitutive relations are often written in terms of the invariants of the left Cauchy-Green deformation tensor defined as $\underline{\mathbf{B}}=\underline{\mathbf{F}} \underline{\mathbf{F}}^{T}=\underline{\mathbf{B}}^{T}$ [19]. Since the swelling pre-deforms the material we introduce the left Cauchy-Green deformation tensor for each network $i$, denoted by $\underline{\mathbf{B}}_{N}^{i}$, which captures both swelling and post-swelling deformation:

$$
\underline{\mathbf{B}}_{N}^{i}=\underline{\mathbf{F}}_{N}^{i}\left(\underline{\mathbf{F}}_{N}^{i}\right)^{T}=\underline{\mathbf{F}}_{(N-1)}^{i}\left(\underline{\mathbf{F}}_{(N-1)}^{i}\right)^{T}=\left(\lambda_{i-1}^{d r y} \prod_{k=i}^{N-1} \lambda_{s k}\right)^{2} \underline{\mathbf{F}}^{T}=\left(J_{s(N-1)}^{i}\right)^{2 / 3} \underline{\mathbf{B}}
$$

The first, second and third invariants of $\underline{\mathbf{B}}_{N}^{i}$ are respectively given by

\begin{tabular}{|l|l|}
\hline$I_{1}\left(\underline{\mathbf{B}}_{N}^{i}\right)=\operatorname{tr}\left(\underline{\mathbf{B}}_{N}^{i}\right)=\left(J_{s(N-1)}^{i}\right)^{2 / 3} \operatorname{tr}(\underline{\mathbf{B}})$ & (10) \\
\hline$I_{2}\left(\underline{\mathbf{B}}_{N}^{i}\right)=\frac{1}{2}\left[\left(\operatorname{tr}\left(\underline{\mathbf{B}}_{N}^{i}\right)\right)^{2}-\operatorname{tr}\left(\left(\underline{\mathbf{B}}_{N}^{i}\right)^{2}\right)\right]=\frac{\left(J_{s(N-1)}^{i}\right)^{4 / 3}}{2}\left[(\operatorname{tr}(\underline{\mathbf{B}}))^{2}-\operatorname{tr}\left(\underline{\mathbf{B}}^{2}\right)\right]$ & \\
\hline and
\end{tabular}

$$
I_{3}\left(\underline{\mathbf{B}}_{N}^{i}\right)=\operatorname{det}\left(\underline{\mathbf{B}}_{N}^{i}\right)=\left(J_{N}^{i}\right)^{2}
$$

where

$$
J_{N}^{i}=\operatorname{det}\left(\underline{\mathbf{F}}_{N}^{i}\right)=\operatorname{det}\left(\underline{\mathbf{\Phi}}_{(N-1)}^{i}\right) \operatorname{det}(\underline{\mathbf{F}})=J_{s(N-1)}^{i} \operatorname{det}(\underline{\mathbf{F}})
$$

In many cases the deformation of the completed MNE can be assumed to be incompressible so that $\operatorname{det}(\underline{\mathbf{F}})=1$. In this case $J_{N}^{i}=J_{s(N-1)}^{i}$. 


\subsection{Constitutive Model}

The total strain energy per unit volume of the MNE is assumed to be the sum of the contributions from each network $\left(U_{N}^{i}\left(\underline{\mathbf{B}}_{N}^{i}\right)\right)$ weighted by its volume fraction $\left(\phi_{N}^{i}\right)$, or

$$
U_{N}(\underline{\mathbf{B}})=\sum_{i=1}^{N} \phi_{N}^{i} U_{N}^{i}\left(\underline{\mathbf{B}}_{N}^{i}\right)
$$

If we assume that the completed MNE is incompressible under further deformation, the Cauchy stress can be computed as follows (see Supporting Information Section S2)

$$
\underline{\boldsymbol{\sigma}}_{N}=\sum_{i=1}^{N} \underline{\boldsymbol{\sigma}}_{N}^{i}=\sum_{i=1}^{N} \phi_{N}^{i} 2 \frac{\partial U_{N}^{i}\left(\underline{\mathbf{B}}_{N}^{i}\right)}{\partial \underline{\mathbf{B}}_{N}^{i}} \underline{\mathbf{B}}_{N}^{i}-p \underline{\mathbf{I}}
$$

where $p$ is a Lagrange multiplier used to satisfy incompressibility and $\underline{\boldsymbol{\sigma}}_{N}^{i}$ represents the contribution to the Cauchy stress from network $i$ and is given by

$$
\underline{\boldsymbol{\sigma}}_{N}^{i}=\phi_{N}^{i} 2 \frac{\partial U_{N}^{i}\left(\underline{\mathbf{B}}_{N}^{i}\right)}{\partial \underline{\mathbf{B}}_{N}^{i}} \underline{\mathbf{B}}_{N}^{i}-p^{i} \underline{\mathbf{I}}
$$

Here $p=\sum_{i=1}^{N} p^{i}$ and $p^{i}$ is the contribution to the Lagrange multiplier from network $i$. Note that the determination of $p$ using boundary conditions must be based on $\underline{\boldsymbol{\sigma}}_{N}$ and not $\underline{\boldsymbol{\sigma}}_{N}^{i}$. For the case of uniaxial extension, it is possible to separate $p$ into contributions $p^{i}$ from each network.

To complete the constitutive relation for the MNE, it is necessary to specify the strain energy density function $U_{N}^{i}\left(\underline{\mathbf{B}}_{N}^{i}\right)$ for each network. Since the properties of the first (and filler) network usually 
differ significantly from the matrix networks, below we propose two different formulations of $U_{N}^{i}\left(\underline{\mathbf{B}}_{N}^{i}\right)$ for the filler and matrix networks.

\subsubsection{Filler Network}

The filler network contains a relatively high density of crosslinks. Also, based on experimental measurements with photophores [13] many chains in this network can be ruptured in the bulk material before crack propagation. Therefore, a constitutive model is needed to capture the nonlinear response of polymer chains as they are stretched and ultimately break. In addition, because damage was observed over a range of strains, the model must contain a feature which allows the chains to reach critical extensions and break at different times. This has been accomplished in several ways in the literature: polydispersity or a distribution of chain lengths [20] results in shorter chains breaking before longer chains; a distribution of initial end-to-end distance of the chains results in initially more stretched chains breaking before more coiled chains [21]; and different orientations of the chains relative to the principal deformation direction lead to chains aligned with the deformation breaking before unaligned chains $[22,23]$. Although in real materials all the previously mentioned effects may be present, we will focus on polydispersity of chain lengths. It should be noted that in this work the distribution of chain lengths is estimated based on experimental measurements of chain rupture in Section 4, which effectively captures all effects that contribute to chain breakage at different extensions. Based on the above considerations, for the filler network we propose a strain energy potential in the following form

$$
U_{N}^{1}=\mu \int_{1}^{\infty} f\left(N_{K}\right) b\left(r_{\max }^{*}, N_{K}\right) N_{K} E_{c h}^{*}\left(r^{*}\right) d N_{K},
$$

where, $\mu=v k_{B} T, v$ is volumetric density of chains (before any swelling), $k_{B}$ is the Boltzmann constant, $T$ is the absolute temperature, $N_{K}$ is the number of Kuhn segments in a polymer chain 
between crosslinks (i.e. representing the chain length), and $f\left(N_{K}\right)$ is a probability density function which describes the distribution of chain lengths and satisfies $\int_{1}^{\infty} f\left(N_{K}\right) d N_{K}=1 . r^{*}=r / N_{K} A_{o}$ is the fractional chain extension, where $r$ is the end-to-end distance of the polymer chain and $A_{o}$ is the Kuhn length of the polymer chain. $b\left(r_{\max }^{*}, N_{K}\right)$ are a set of damage functions that depend on the chainlength $\left(N_{K}\right)$ and will be described later. $r_{\max }^{*}$ is the maximum fractional extension a chain has reached in its deformation history, and $E_{c h}^{*}\left(r^{*}\right)=E_{c h}\left(r^{*}\right) / k_{B} T$ is the nondimensional chain energy to be discussed next. The integration in Eq. (17) accounts for the total contribution of all chain lengths to the strain energy. More details on the derivation of this type of strain energy potential can be found in [20].

Using the "8-chain" model by Arruda and Boyce [24], the following expression can be written to relate the chain extension $r^{*}$ to $I_{1}\left(\underline{B}_{N}^{1}\right)$

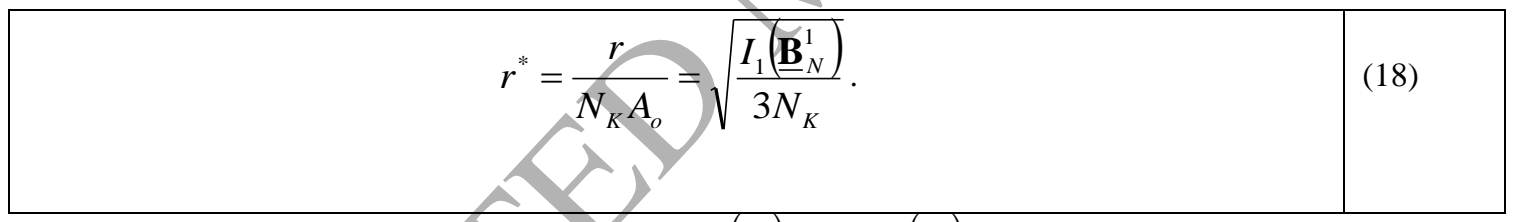

Introducing a dimensionless chain force as $F_{c h}^{*}\left(r^{*}\right)=A_{o} F_{c h}\left(r^{*}\right) / k_{B} T$, the dimensionless polymer chain energy needed in Eq. (17) is calculated from

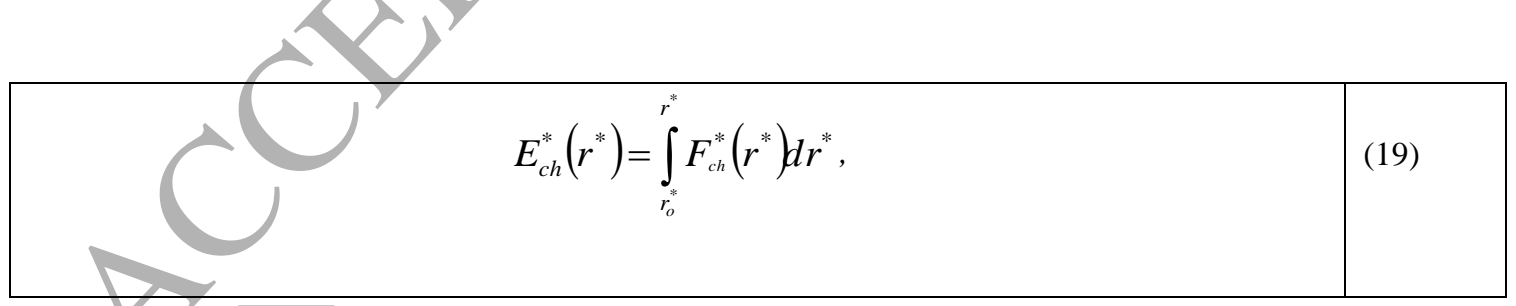
where $r_{o}^{*}=\sqrt{1 / N_{K}}$ is the fractional extension of the chain under load-free condition. We have chosen to start this integration at $r_{o}^{*}$ rather than zero to enforce the zero-strain energy condition at the relaxed 
state where $I_{1}\left(\underline{\mathbf{B}}_{N}^{1}\right)=3$; this choice introduces a constant into the strain energy density and has no impact on the stress predicted by the model.

The kinematic assumption (Eq. (18)) implies that the strain energy density depends only on $I_{1}\left(\underline{\mathbf{B}}_{N}^{1}\right)$. One may argue that during swelling and drying there is a large volume change which implies the third invariant $I_{3}\left(\underline{\mathbf{B}}_{N}^{1}\right)$ changes, and for this reason the strain energy can also depend on $I_{3}\left(\underline{\mathbf{B}}_{N}^{1}\right)$. In fact, such a term has often been included in the strain energy function when modeling swelling and drying. For example, Hong et al. include a term $\mu \ln \left(\sqrt{I_{3}\left(\underline{B}_{N}^{1}\right)}\right)$ for the filler network [25] when modeling gels. On the other hand, this term has been the subject of some controversy in the literature [26] and the discussion remains inconclusive [27]. Nevertheless, the effect of such a term is shown to be rather small in most practical cases [27]. Furthermore, in this work we focus on the deformation of a pre-swollen MNE that remains incompressible during post-swelling and drying deformation, in which case $I_{3}\left(\underline{\mathbf{B}}_{N}^{1}\right)$ will be constant, and whether the $I_{3}\left(\underline{\mathbf{B}}_{N}^{1}\right)$ dependency is included in the strain energy density function will not impact our results.

Since $U_{N}^{1}$ depends only on $I_{1}\left(\underline{\mathbf{B}}_{N}^{1}\right)$, we can write

$$
\frac{\partial U_{N}^{1}\left(I_{1}\left(\underline{\mathbf{B}}_{N}^{1}\right)\right)}{\partial \underline{\mathbf{B}}_{N}^{1}}=\frac{\left.d U_{N}^{1}\left(I_{1}\left(\underline{\mathbf{B}}_{N}^{1}\right)\right)\right)}{d I_{1}\left(\underline{\mathbf{B}}_{N}^{1}\right)} \frac{\partial I_{1}\left(\underline{\mathbf{B}}_{N}^{1}\right)}{\partial \underline{\mathbf{B}}_{N}^{1}}=\frac{d U_{N}^{1}\left(I_{1}\left(\underline{\mathbf{B}}_{N}^{1}\right)\right)}{d I_{1}\left(\underline{\mathbf{B}}_{N}^{1}\right)} \underline{\mathbf{I}}
$$

Thus, to evaluate the stress in Eq. (15) the derivative of the strain energy potential is needed, which can be calculated by

$$
\frac{d U_{N}^{1}\left(I_{1}\left(\underline{\mathbf{B}}_{N}^{1}\right)\right)}{d I_{1}\left(\underline{\mathbf{B}}_{N}^{1}\right)}=\frac{\mu}{6} \int_{1}^{\infty} \frac{f\left(N_{K}\right) b\left(r_{\max }^{*}, N_{K}\right) F_{c h}^{*}\left(r^{*}\right)}{r^{*}} d N_{K} .
$$


There are several models in the literature which can be used to obtain $F_{c h}^{*}\left(r^{*}\right)$ or $E_{c h}^{*}\left(r^{*}\right)$ [24,28,29]; one of the most commonly used models is the Arruda-Boyce model [24]. However, the $F_{c h}^{*}\left(r^{*}\right)$ relationship adopted in the Arruda-Boyce model is based on a freely jointed chain model with Langevin statistics, where the chain is inextensible and there is a singularity in chain force when $r^{*}=1$. This singular behavior allows only a very small fraction of the chains to experience large forces at any time, which limits the maximum stress that is obtainable. On the contrary, the novel microstructure of the MNE leverages prestretch via swelling to create many chains in the filler network that are subjected to large tensile forces and can rupture during the deformation. Theselarge tensile forces are sufficient to deform bonds and elongate chains, as discussed in a recent work [30]. Therefore, the Arruda-Boyce or any inextensible model based purely on entropic elasticity may not be suitable for this type of material. In a work to be reported separately, we incorporated bond deformation into the chains' backbone, and obtained a force-extension relationship by minimizing the free energy of a polymer chain consisting of configurational entropy and bond deformation energy. Compared with entropic chain models, this new relationship has been shown to provide better agreement with single chain extension data in experiments, especially when $r^{*}>0.9$ [31]. For the poly ethyl-acrylate (PEA) chain relevant to the present work, the force-extension relationship can be approximated in closed form by polynomial functions as follows

\begin{tabular}{|cc|c|c|c|c|c|}
$F_{c h}^{*}=\frac{1}{2}\left(1-r^{*}\right)^{-2}-\frac{1}{2}-2 r^{*}$ & $r^{*}<0.9$ \\
$F_{c h}^{*}=\frac{513}{20}+501\left(r^{*}-0.9\right)+26238\left(r^{*}-0.9\right)^{2}+68436\left(r^{*}-0.9\right)^{3}$ & $r^{*} \geq 0.9$ & (21)
\end{tabular}

Last, we comment on the damage functions $b$, which represent the fractions of chains that remain intact and are dependent on the number of Kuhn lengths $N_{K}$ in the chain. $b$ equals 1 for 
undamaged material and decreases as chains are ruptured. The rupture of polymer chains can be modeled using rate dependent nonlinear ordinary differential equations $[20,28]$ where the rate of chain scission depends on the force acting on the bonds in the polymer chain. On the other hand, it was found that [20] if the deformation speed was sufficiently high the damage was nearly rate independent. Extension rates used in the experiment of Ducrot et al fall into this regime and the material exhibited negligible rate dependence [13]. In this work, we focus on this regime and approximate the damage functions by rate-independent functions in the form of $b\left(r_{\max }^{*}, N_{K}\right)$. These damage functions have been obtained in [31] by numerically solving the rate equations at a given stretch rate in the rate-independent regime, and they are summarized in Supporting Information Section S3. Since the chain rupture is irreversible, during loading (as $r_{\max }^{*}$ increases) $b$ decreases, whereas upon unloading when the chain end-to-end distance $r^{*}$ becomes smaller than $r_{\max }^{*}$, the value of $b$ cannot increase.

\subsubsection{Matrix Networks}

When the matrix networks $(i>1)$ are formed the concentration of the crosslinker used is approximately 145 times less than that in the filler network [13]. This results in much longer chain lengths between crosslinks in the matrix networks. Since the chains in these networks have less (or even no) prestretch compared with the chains in the filler network and hence do not experience large fractional extensions, it is reasonable to assume that they remain intact (i.e. no rupture) during deformation and their strain stiffening is insignificant. With this consideration, a neo-Hookean material model, which is based on Gaussian freely jointed chains with a linear force extension relationship [27], would seem to be a good choice to represent the matrix networks. However, because these networks are so lightly crosslinked, physical entanglements create a strain softening effect at small deformations [13] which cannot be captured by the neo-Hookean model. This softening effect is well described by a 
molecular model by Rubinstein and Panyukov [32], but it is not straightforward to convert the molecular model into a continuum model for general 3D deformations. Instead, we will use the generalized neoHookean constitutive model [16] for the individual matrix networks to phenomenologically capture the softening effect:

$$
U_{N}^{i}\left(I_{1}\left(\underline{\mathbf{B}}_{N}^{i}\right)\right)=\frac{\mu_{M}}{2 n_{M}}\left\{\left[1+\left(I_{1}\left(\underline{\mathbf{B}}_{N}^{i}\right)-3\right)\right]^{n_{M}}-1\right\}, i>1
$$

where $\mu_{M}$ is the shear modulus and $n_{M}$ is an additional parameter which controls the shape of $U_{N}^{i}\left(I_{1}\left(\underline{\mathbf{B}}_{N}^{i}\right)\right)$. Note that a $3^{\text {rd }}$ parameter from the generalized neo-Hookean model [16] was omitted to minimize the number of parameters that are introduced into the MNE model. Like the filler network, the strain energy for each matrix network depends only on the first invariant, i.e. $I_{1}\left(\underline{\mathbf{B}}_{N}^{i}\right)$, thus as before to evaluate the stress in Eq. (15) the derivative of the strain energy potential is obtained

$$
\frac{d U_{N}^{i}\left(I_{1}\left(\underline{\mathbf{B}}_{N}^{i}\right)\right)}{d I_{1}\left(\underline{\mathbf{B}}_{N}^{i}\right)}=\frac{\mu_{M}}{2}\left[1+\left(I_{1}\left(\underline{\mathbf{B}}_{N}^{i}\right)-3\right)\right]^{n_{M}-1}, i>1 .
$$

\section{Application of the Model}

Below we will use the model established above to predict the mechanical response of MNEs under uniaxial loading conditions and compare the results with experimental data. Assuming that the networks contribute independently to the total stress, parameters pertaining to the matrix networks will be extracted from available data for these networks alone. Chain length distribution in the filler network will be estimated based on data for DN and TN elastomers with light emitting photophores embedded in the filler network. Finally, comparison will be made to uniaxial extension and cyclic loading experiments 
conducted [18] for a variety of MNEs (SN, DN and TN) where the prestretch caused by the first swelling and drying was varied.

Before we proceed, it will be beneficial to specialize the general constitutive model for uniaxial extension. The left Cauchy-Green deformation tensor in this case is given by

$$
\underline{\mathbf{B}}=\lambda^{2} \underline{\mathbf{e}}_{1} \otimes \underline{\mathbf{e}}_{1}+\lambda^{-1} \underline{\mathbf{e}}_{2} \otimes \underline{\mathbf{e}}_{2}+\lambda^{-1} \underline{\mathbf{e}}_{3} \otimes \underline{\mathbf{e}}_{3},
$$

where $\lambda$ is the stretch of the sample and $\underline{\mathbf{e}}_{1}, \underline{\mathbf{e}}_{2}, \underline{\mathbf{e}}_{3}$ are orthogonal unit vectors forming the basis of the deformed configuration. Using Eq. (9) and the fact that the strain energy potentials depend only on $I_{1}\left(\underline{\mathbf{B}}_{N}^{i}\right)$, Eq.(15) can be rewritten as follows

$$
\underline{\boldsymbol{\sigma}}_{N}=2\left[\sum_{i=1}^{N} \phi_{N}^{i}\left(J_{s(N-1)}^{i}\right)^{2 / 3} \frac{d U_{N}^{i}\left(I_{1}\left(\underline{\mathbf{B}}_{N}^{i}\right)\right)}{d I_{1}\left(\underline{\mathbf{B}}_{N}^{i}\right)}\right] \underline{\mathbf{B}}^{-} p \underline{\mathbf{I}}
$$

In uniaxial extension, $\underline{\boldsymbol{\sigma}}_{N}$ only has one nonzero component which we denote as $\sigma_{N}$. Requiring the other stress components to be zero gives the Lagrange multiplier $p$. Substituting Eq. (24) into Eq. (25), we can obtain an expression for $\sigma_{N}$

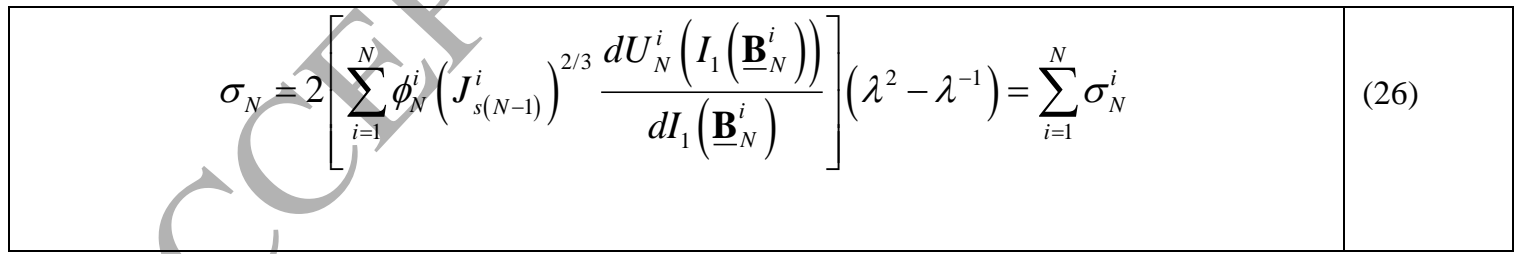

where the stress contribution from each network is given by

$$
\sigma_{N}^{i}=\phi_{N}^{i} 2\left(J_{s(N-1)}^{i}\right)^{2 / 3} \frac{d U_{N}^{i}\left(I_{1}\left(\underline{\mathbf{B}}_{N}^{i}\right)\right)}{d I_{1}\left(\underline{\mathbf{B}}_{N}^{i}\right)}\left(\lambda^{2}-\lambda^{-1}\right) .
$$


Note that a portion of the Lagrange multiplier is assigned to each network so that the stress in each network is zero in the MNE's reference configuration which is equivalent to satisfying the traction-free boundary condition on the lateral surface of the tensile sample for each network. As such the stress $\sigma_{N}^{i}$ is the contribution of each network to the total uniaxial tensile stress, instead of the actual stress sustained in each network.

We will extensively compare predictions from the model with experimental data, which without processing, will be the nominal (engineering) stress. For uniaxial extension, the engineering stress can be related to the Cauchy stress (or true stress) as follows

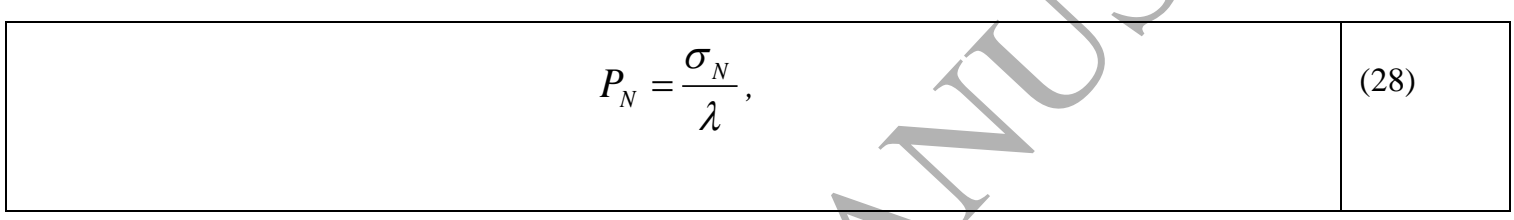

similarly, $P_{N}^{i}=\sigma_{N}^{i} / \lambda$

\subsection{Extracting Parameters for the Matrix Network}

Experiments were conducted on the matrix networks alone to determine the parameters $\mu_{M}$ and $n_{M}$ in Eq. (22). The stress for a single matrix network alone, $P_{M}$, can be evaluated from Eqs. (26) and (28) by setting $N=2, \phi_{2}^{1}=0$, and $\phi_{2}^{2}=1$. The experimental data and the best fit are shown in Figure 3. Since these experiments were done for a dry matrix network, when applying Eq. (22) to the $\mathrm{MNE}, \mu_{M}$ is replaced by

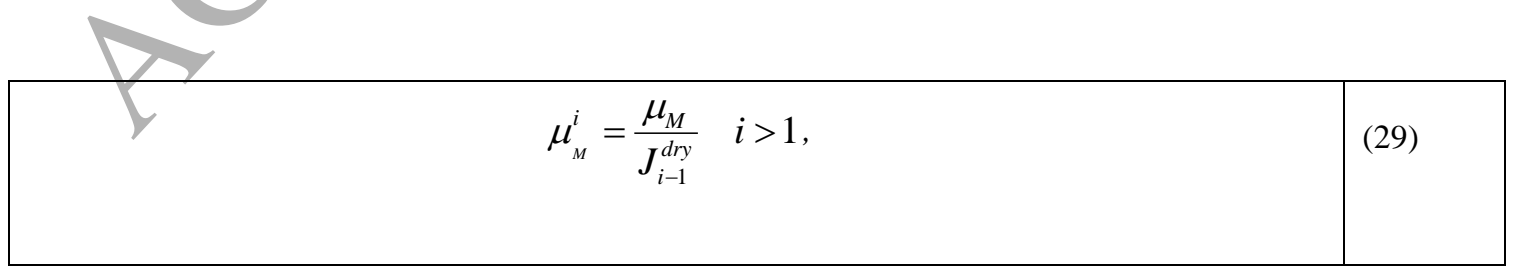

where $\mu_{M}^{i}$ is the shear modulus for network $i$ in its reference state. 


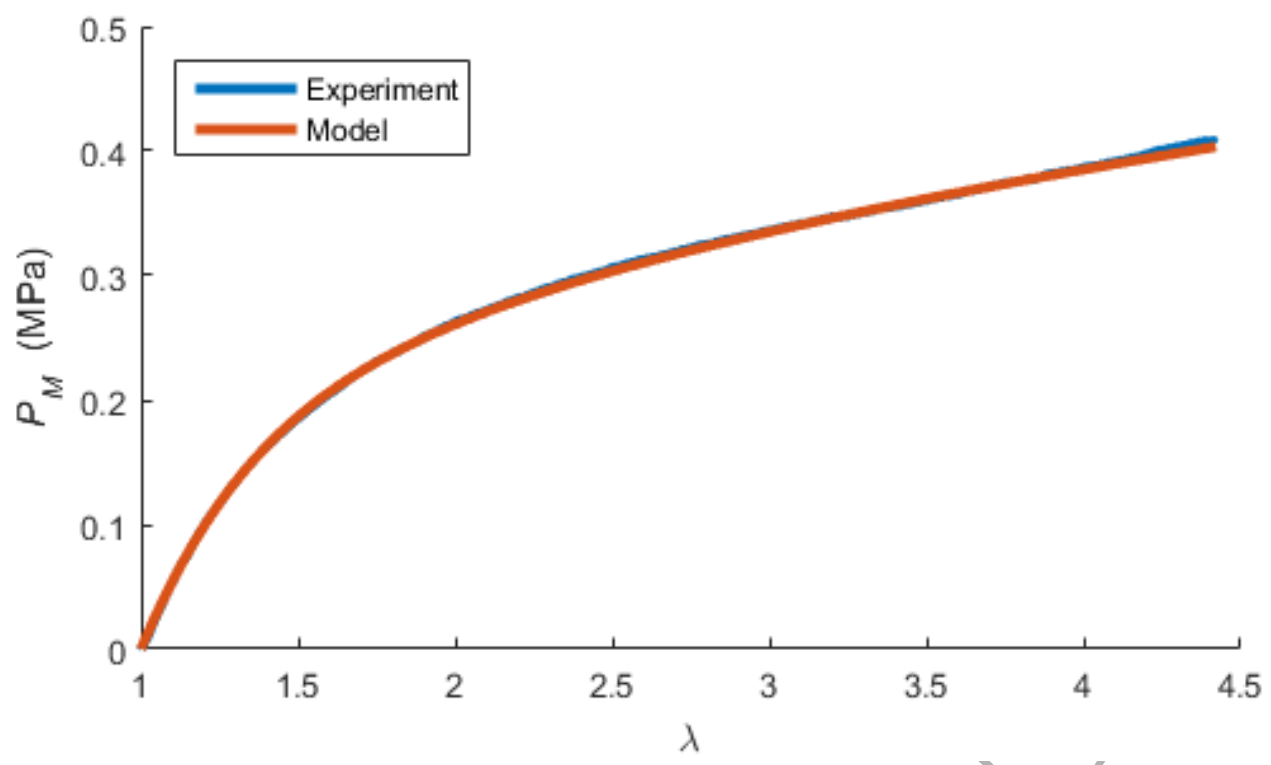

Figure 3: Engineering stress plotted against stretch for uniaxial extension of the matrix network. The model with parameters $n_{M}=0.8325$ and $\mu_{M}=0.2 \mathrm{MPa}$ was found to provide a good fit to the experimental data. 


\subsection{Distribution of Chain Lengths in Filler Network}

Several specialized experiments were conducted for MNEs where photophores were used to crosslink chains in the filler network [13]. These photophores emit light when they rupture, and the recorded light intensity provides a means to estimate the distribution of polymer chain lengths in the filler network. Specifically, when photophores are added to the chains they create weaker links. If we assume that the rate of chain rupture is proportional to the rate of photophore rupture, the chain length distribution can be correlated to the measured light emission data.

To see this, note that the recorded light intensity was integrated over the sample [13]. Assuming that the elastomer is transparent so light produced by scission throughout the volume can all be collected, the light emission intensity can be expressed as

$$
L I=-\int_{A} \alpha_{l} v \int_{1}^{\infty} f\left(N_{K}\right) \frac{d b\left(r_{\max }^{*}, N_{K}\right)}{d t} d N_{K} D_{N} d S,
$$

where $\alpha_{l}$ is a proportionality constant which relates light intensity to photophore rupture, $v$ is the volumetric density of chains which is proportional to the density of photophores, $t$ is time, $D_{N}$ is the thickness of the sample after the $(N-1)^{\text {th }}$ swelling and drying, and $S$ is the area of the sample perpendicular to the thickness direction. Under homogeneous deformation during uniaxial extension, Eq. (30) can be rewritten as follows

$$
L I=\beta_{l} \int_{1}^{\infty} f\left(N_{K}\right) \frac{d b\left(r_{\max }^{*}, N_{K}\right)}{d t} d N_{K}
$$


where $\beta_{l}=-\alpha_{l} v V_{N}$ and $V_{N}=D_{N} S$ is the constant volume of the sample. To evaluate $d b\left(r^{*}\right) / d t$, consider

\begin{tabular}{|c|}
\hline$\frac{d b}{d t}=\frac{d b}{d r_{\max }^{*}} \frac{d r_{\max }^{*}}{d I_{1}\left(\underline{\mathbf{B}}_{N}^{1}\right)} \frac{d I_{1}\left(\underline{\mathbf{B}}_{N}^{1}\right)}{d \lambda} \dot{\lambda}=\frac{d b}{d r_{\max }^{*}} \frac{r_{\max }^{*}}{2 I_{1}(\underline{\mathbf{B}})}\left(2 \lambda-2 \lambda^{-2}\right) \dot{\lambda}$, \\
where $\frac{d r_{\max }^{*}}{d I_{1}\left(\underline{\mathbf{B}}_{N}^{1}\right)}=\frac{r_{\max }^{*}}{2 I_{1}\left(\underline{\mathbf{B}}_{N}^{1}\right)}=\frac{r_{\max }^{*}}{2\left(J_{s(N-1)}^{1}\right)^{2 / 3} I_{1}(\underline{\mathbf{B}})}$ and $\frac{d I_{1}\left(\underline{\mathbf{B}}_{N}^{1}\right)}{d \lambda}=\left(J_{s(N-1)}^{1}\right)^{2 / 3}\left(2 \lambda-2 \lambda^{-2}\right)$ are respectively
\end{tabular} obtained from Eq. (18) and Eqs. (10) and (24), $d b / d r_{\max }^{*}$ is known based on the damage functions presented in Supporting Information Section S3, and $\dot{\lambda}$ is the stretching rate prescribed in the experiment. If $r^{*}$ does not exceed its previous maximum value, no additional damage will occur and Eq. (32) should be taken to be zero.

In general, the rupture of bonds follows certain kinetics and will not occur at the same extension even for chains of the same length. However, the damage will be concentrated near the vicinity of highest extension. This allows us to simplify Eq. (32) by approximating $d b / d r_{\max }^{*}=-\delta\left(r_{\max }^{*}-r_{p k}^{*}\right)$ where $\delta$ is the Dirac delta distribution and $r_{p k}^{*}$ is the extension at which the peak value of $d b / d r_{\max }^{*}$ occurs (see Supporting Information Section S3). Introducing this approximation and Eq. (32) into Eq. (31) gives 
$L I=-\beta_{l} \frac{\left(\lambda-\lambda^{-2}\right)}{\left(\lambda^{2}-2 \lambda^{-1}\right)} \dot{\lambda} \int_{1}^{\infty} f\left(N_{K}\right) \delta\left(r_{\max }^{*}-r_{p k}^{*}\right) r_{\text {max }}^{*} d N_{K}=-\beta_{l} \frac{\left(\lambda-\lambda^{-2}\right)}{\left(\lambda^{2}+2 \lambda^{-1}\right)} \dot{\lambda} f\left(N_{K}\left(r_{p k}^{*}\right)\right) r_{p k}^{*}$,

which can be rearranged to solve for $f\left(N_{K}\left(r_{p k}^{*}\right)\right)$

$$
f\left(N_{K}\left(r_{p k}^{*}\right)\right)=\frac{L I(\lambda)}{-\beta_{l} r_{p k}^{*}} \frac{\left(\lambda^{2}+2 \lambda^{-1}\right)}{\left(\lambda-\lambda^{-2}\right)},
$$

where $N_{K}\left(r_{p k}^{*}\right)=I_{1}\left(\underline{\mathbf{B}}_{N}^{1}\right) / 3 r_{p k}^{* 2}$ using Eq. (18). Based on Eq. (33), the experimentally measured light emission data was converted into a chain length distribution, as shown in Figure 4. A probability distribution function in the form of Eq. (34) was found to provide a good fit for the chain length distribution measured from light emission data (see Figure 4)

$$
f\left(N_{K}\right)=A_{f}\left(N_{K}-N_{\text {min }}\right)^{a_{f}} \exp \left(-b_{f}\left(N_{K}-N_{\text {min }}\right)\right) \begin{aligned}
& N_{K} \geq N_{\text {min }}, \\
& N_{K}<N_{\text {min }}
\end{aligned},
$$

where $N_{\min }$ is the minimum number of load bearing Kuhn segments present in the material, $a_{f}$ and $b_{f}$

control the shape of the probability function, and $A_{f}$ is used to ensure the normalization requirement

$\int_{1}^{\infty} f\left(N_{K}\right) d N_{K}=1$ is satisfied. $a_{f,}, b_{f}$, and $N_{\text {min }}$ are the three parameters extracted from the fitting. In

Figure 4, light emission data are not available for large chain lengths since the material already failed, so the fitting curve is a smooth extrapolation of the measured data. 


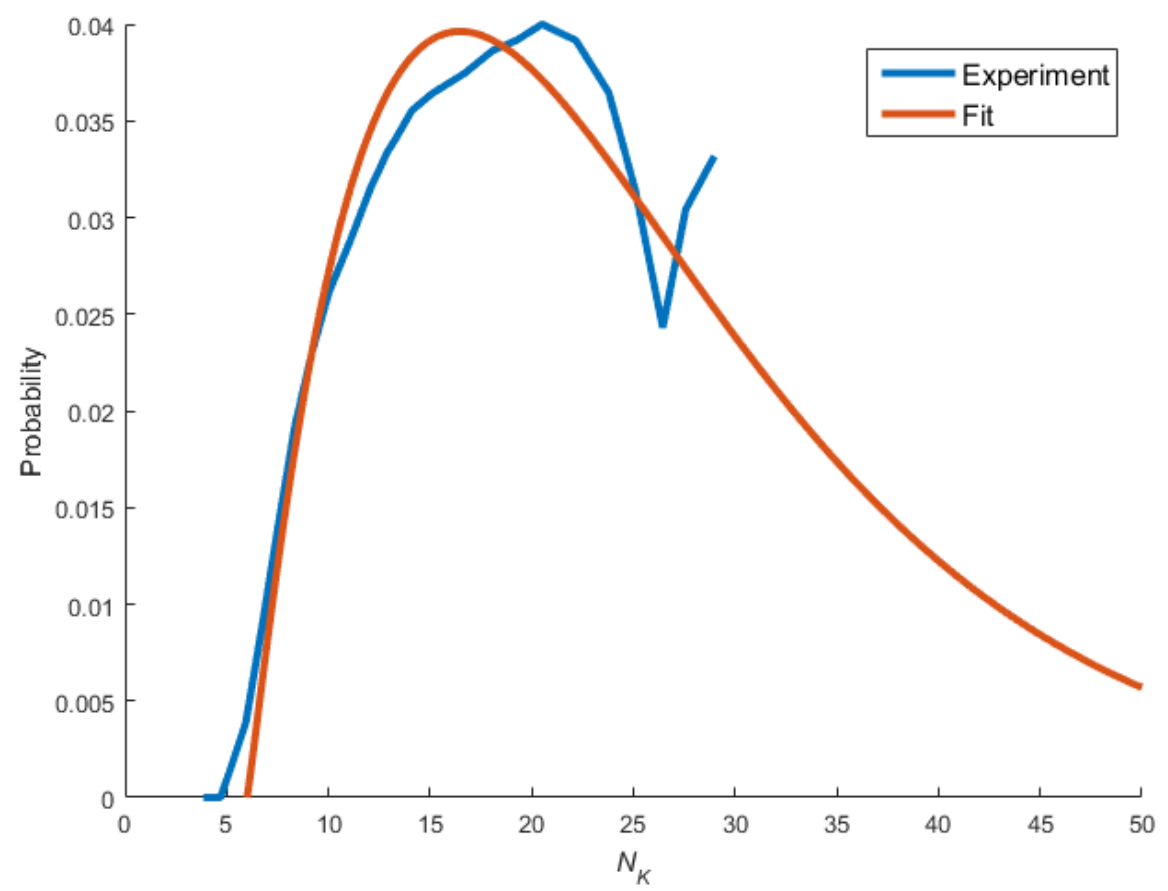

Figure 4: Chain length distribution estimated by fitting light emission data. Light emission experimental data from Ducrot et al [13] are converted to chain length probability using Eq.(33) where $\beta_{l}=2.7 \times 10^{9}$ was found to provide a good fit. $r_{p k}^{*} \approx 1.03$, and for a $1 \mathrm{~mm} . \mathrm{s}^{-1}$ crosshead velocity the stretch rate for the centralpart of the sample was estimated to be $\dot{\lambda}=0.08 \mathrm{~s}^{-1}$. The chain length distribution can be approximated by Eq. (34) with $N_{\min }=6, a_{f}=1.1, b_{f}=0.105$.

\subsection{Uniaxial Extension of MNE}

Model prediction will be compared with two groups of experiments: MNEs with and without photophore. The chain length distribution from Figure 4 was applied directly to the MNE with photophore. Whereas for the MNE without photophore, the same form of distribution (Eq. (34)) was used but the parameters $N_{\min }, a_{f}$ and $b_{f}$ were slightly different from those in Figure 4 . The same tuned distribution was used for all the experiments without photophore, which involved several data sets with different pre-stretches. 


\subsubsection{With Photophore}

For the experiments on MNEs with photophores [13], i.e., DNP and TNP from Table 1, the prestretches of the networks are $\lambda_{s 1}=1.48$ and $\lambda_{s 1} \lambda_{s 2}=2.72$, respectively. $\mu$ in Eq. (17) is related to the volumetric density of chains in the filler network before any swelling, and is a fitting parameter since no SN data was available for MNE with photophores. When used with the chain length distribution in Figure 4, it was found that $\mu=0.264 M P a$ and a slightly adjusted value of $\lambda_{s 1}=1.54$ provided the best fit to the experimental data for both DN and TN elastomers For TN $\lambda_{s 1} \lambda_{s 2}=2.72$ was held fixed so that $\lambda_{s 2}=1.77$. The comparisons are shown in Figure 5 , where the model reproduces all the key features observed in the DN and TN data. For example, the model correctly predicts the small initial slope observed in the stress-stretch curve for both DN and TN; the slope is small in this region because no chains are sufficiently stretched to create large forces. With further stretching the shortest chains become highly stretched to cause an upturn in the stress, which is observed in both the model and the experimental data. Finally, for the TN with further stretching, damage accumulates and causes a second inflection point after which the stress-stretch curve levels off. This phenomenon is not observed in the DN because the material fails before sufficient damage accumulation.

It is of interest to calculate the extension of individual chains under the bulk deformation. Because there is a distribution of different chain lengths in the filler network, these chains are subjected to different extensions at the same bulk deformation. The chain extension as a function of the number of Kuhn segments (i.e. chain length) are shown in Figure 6, for the chains in the filler network of the SN, DN and TN elastomers. Results are shown for two different bulk stretches: $\lambda=1$, corresponding to undeformed elastomers, and $\lambda=2$. Both swelling and drying (from SN to DN and then to TN) and additional bulk deformation (from $\lambda=1$ to $\lambda=2$ ) increase the fractional extension, and their impacts 
are more significant for chains with shorter length. For the TN with $\lambda=2$, large fractional extensions for small $N_{K}$ have caused material damage. To quantify the damage, we define the following quantity

$$
b_{N}=\int_{1}^{\infty} f\left(N_{K}\right) b\left(r_{\max }^{*}, N_{K}\right) d N_{K} \text {, }
$$

which represents the fraction of surviving load bearing chains in the filler network of an MNE with a total of $N$ networks. The evolution of damage for the TN elastomer is presented in Figure 7a). No damage is observed until a sufficient stretch (above 1.5) is reached that causes the shortest chains to break. Afterwards $b_{3}$ steadily decreases with additional stretching as more chains are ruptured. 
a)

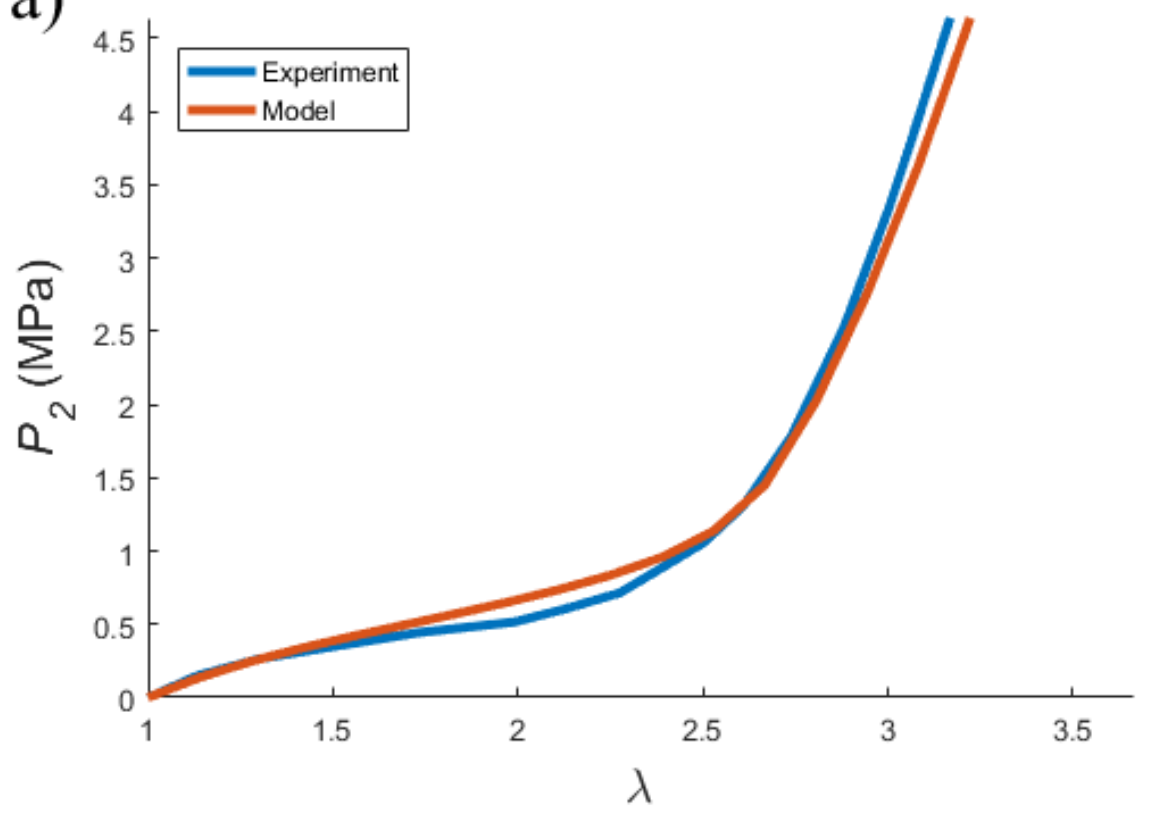

b)

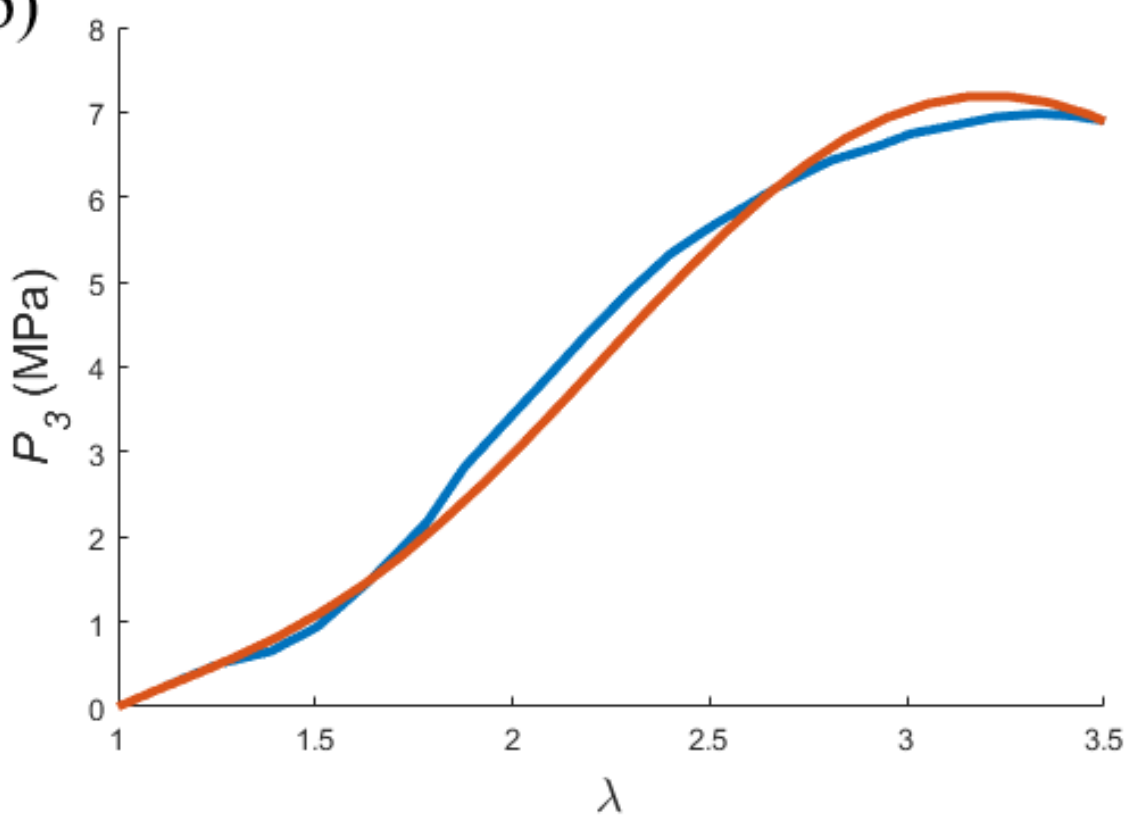


Figure 5 Engineering stress plotted against stretch for uniaxial extension of (a) DNP, (b) TNP. The following prestretches $\lambda_{s 1}=1.54, \lambda_{s 2}=1.77$ and parameter $\mu=0.264 \mathrm{MPa}$ were found to give good agreement with the experimental data. Experimental data from Ducrot et al [13].

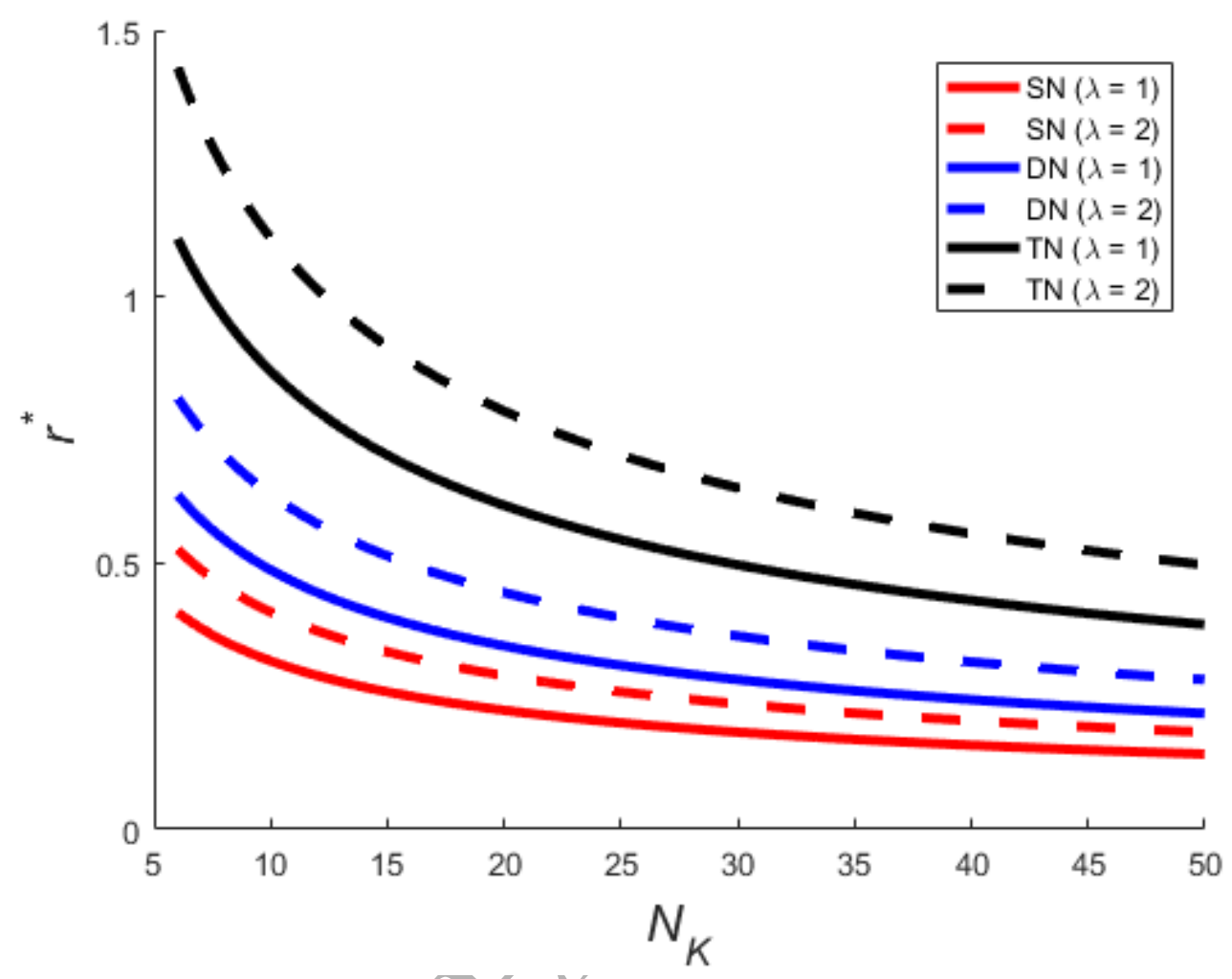

Figure 6 Fractional extension plotted against number of Kuhn segments for individual chains in the filler network of SN, DN and TN elastomers.

Using the two prestretches and Eqs. (5) to (7), the volume fractions of each network can be calculated to be: for the DN elastomer $\phi_{2}^{1}=0.274, \phi_{2}^{2}=0.726$; for the TN elastomer $\phi_{3}^{1}=0.05, \phi_{3}^{2}=0.13$, and $\phi_{3}^{3}=0.82$. The prevailing hypothesis with these materials is that the chains in the filler network $(i=1)$ control the stress whereas the subsequent matrix networks prevent large cracks from forming [13]. Separating the total stress into components from individual constituent networks of the MNE allows us to understand how each network is contributing to the overall property of the MNE. For this purpose, 
the partial stress in each network, as defined by $P_{N}^{i}=\sigma_{N}^{i} / \lambda$ with $\sigma_{N}^{i}$ given by Eq. (27), is plotted in Figure 7b) for the TN elastomer. Here $P_{3}^{1}, P_{3}^{2}$ and $P_{3}^{3}$ represent the equivalent uniaxial tensile stress contributed by the first (and filler), second and third (both matrix) networks, respectively. In agreement with the hypothesis, the filler network provides most of the stress $\left(P_{3}^{1}\right)$, while the contributions of the matrix networks $\left(P_{3}^{2}, P_{3}^{3}\right)$ are almost negligible although they occupy an estimated $94 \%$ of the material volume. 

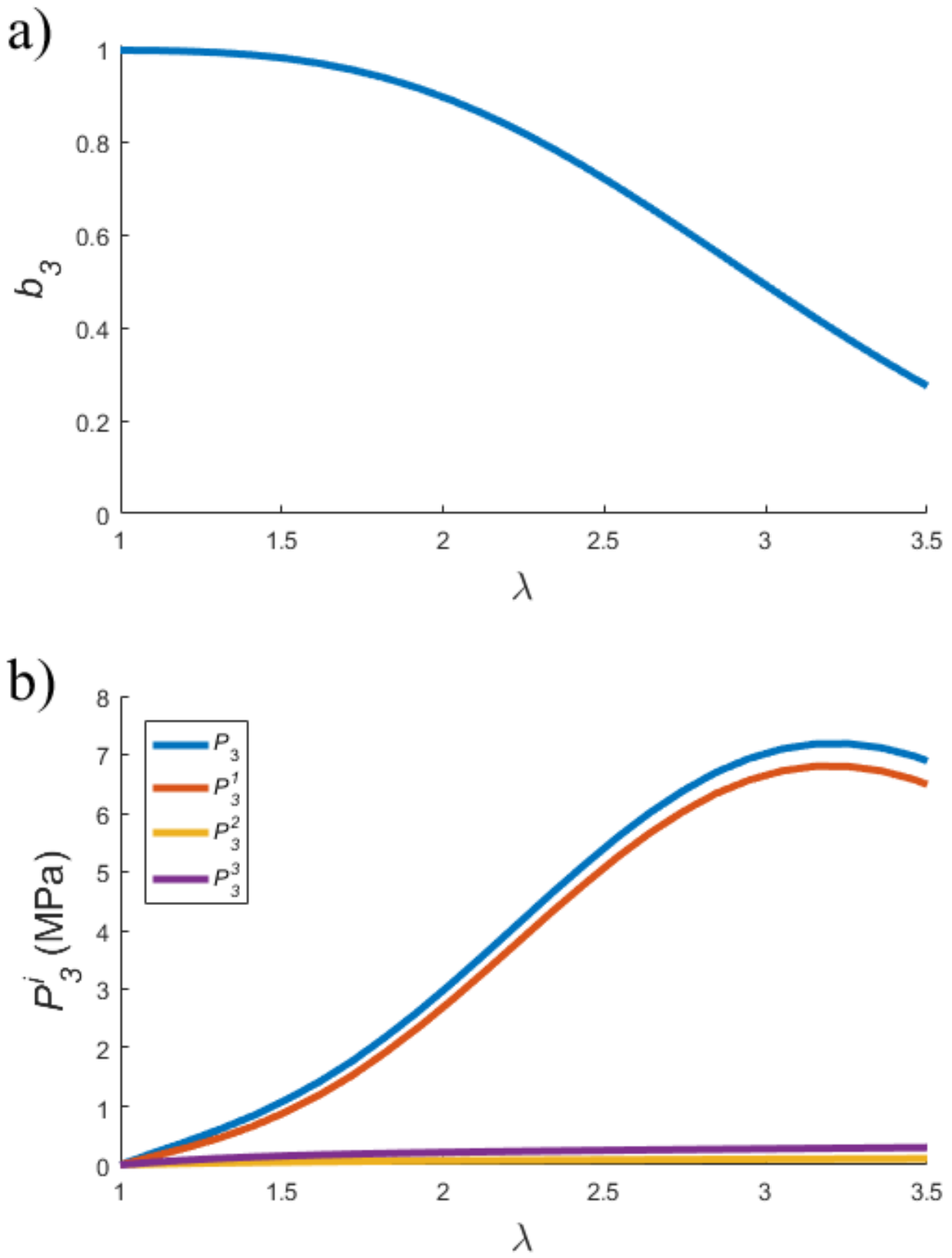

Figure 7: (a) The evolution of damage in the filler network of the TN elastomer is shown by plotting the surviving chain fraction $\left(b_{3}\right)$ against stretch. (b) Contribution from each network to the engineering stress of a TN elastomer under uniaxial extension, plotted against stretch. 


\subsubsection{Without Photophore}

For the MNEs without photophores, we consider three datasets formed from the same original SN but with different prestretches. The stress-stretch curve for the SN is shown in Figure 8, and matching the initial slope provides $\mu=0.215 \mathrm{MPa}$ which will be used for the filler network in all the MNEs. In each case experimental measurements of thickness, percentage of filler network (based on weight), stretch and stress are available. Measurements of sample thickness and weight (Table 2) can be used to estimate the prestretch of the networks. Here \%FN is the percentage of weight of the filler network obtained by weighing the sample before and after swelling and drying. Since the filler and matrix networks are comprised of the same monomers, it is reasonable to assume that all the networks have the same density. This allows the measurement to be directly converted to volume fractions, i.e., $\% \mathrm{FN}=100 \phi_{N}^{1}$, which can then be used to determine $J_{s j}$ using Eqs. (5) and (7). $J_{s j}$ can also be calculated using $J_{s j}=\left(D_{j+1} / D_{j}\right)^{3}=\left(\lambda_{s j}\right)^{3}$, and the values of $\lambda_{s j}$ obtained from the two different approaches can be slightly different, see comparison in Table 3. For this reason, and due to variations observed in samples with the same crosslink density and net swelling (see Supporting Information Section S5), we allow a small amount of tunability in the chosen values of $\lambda_{s j}$, tabulated in Table 3 , to ensure that key features in the mechanical response are properly captured. Finally, other intermediate 
swelling variables calculated from $J_{s 1}, J_{s 2}$ and $\xi_{j}^{m o n}$ are tabulated in Table 4.

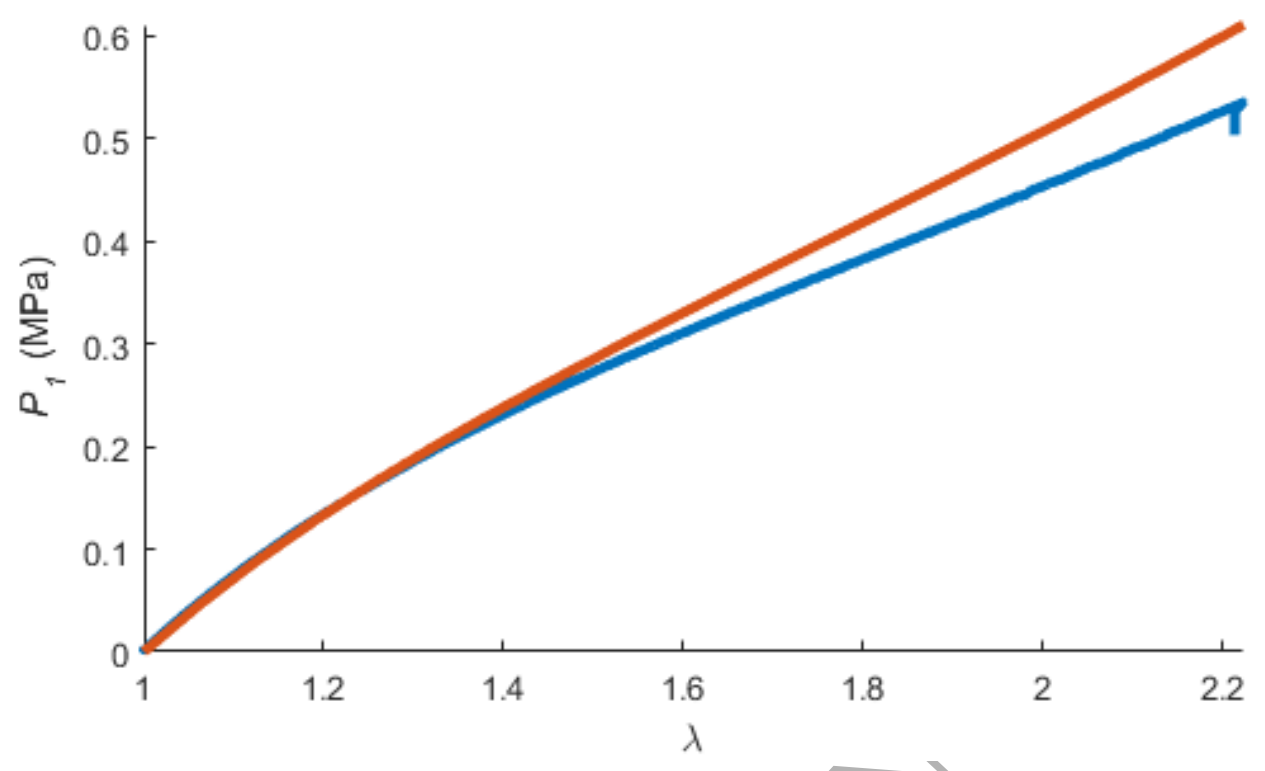

Figure 8: Engineering stress plotted against stretch for uniaxial extension of SN elastomer. Experimental data and model fit (with $\mu=0.215 \mathrm{MPa}$ ) are shown.

Table 2: Swelling data for the 3 datasets.

\begin{tabular}{|l|l|l|l|l|l|l|l|l|}
\hline \multicolumn{2}{|l|}{ Before Swelling } & \multicolumn{2}{l|}{ After 1 ${ }^{\text {st }}$ Swelling and drying } & \multicolumn{2}{l|}{ After 2 ${ }^{\text {nd }}$ Swelling and drying } \\
\hline Sample & $\%$ FN & $\mathrm{D}_{0}(\mathrm{~mm})$ & Sample & $\% \mathrm{FN}$ & $\mathrm{D}_{1}(\mathrm{~mm})$ & Sample & $\% \mathrm{FN}$ & $\mathrm{D}_{2}(\mathrm{~mm})$ \\
& & & & & & & & \\
\hline SN & 100 & 0.74 & $\mathrm{DN} 1$ & 42 & 0.97 & $\mathrm{TN} 1$ & 9.52 & 1.6 \\
\hline SN & 100 & 0.74 & $\mathrm{DN} 2$ & 29.17 & 1.13 & TN2 & 7.39 & 1.79 \\
\hline SN & 100 & 0.74 & DN3 & 20.55 & 1.27 & TN3 & 6.07 & 1.84 \\
\hline
\end{tabular}

Table 3: Swelling ratios estimated by two approaches and the final chosen values. DN and TN in each dataset share the same $J_{s 1}$ values while $J_{s 2}$ values are only applicable to $T N$ samples.

\begin{tabular}{|l|l|l|l|}
\hline Sample & Estimate using $D_{j}$ & Estimate Using \%FN & Value Used \\
\hline
\end{tabular}




\begin{tabular}{|l|r|r|r|r|r|r|}
\hline & \multicolumn{1}{|l|}{$\lambda_{s 1}$} & \multicolumn{1}{|l|}{$\lambda_{s 2}$} & \multicolumn{1}{l|}{$\lambda_{s 1}$} & \multicolumn{1}{l|}{$\lambda_{s 2}$} & \multicolumn{1}{l|}{$\lambda_{s 1}$} & \multicolumn{1}{l|}{$\lambda_{s 2}$} \\
\hline DN1/TN1 & 1.32 & 1.65 & 1.34 & 1.64 & 1.34 & 1.60 \\
\hline DN2/TN2 & 1.51 & 1.58 & 1.51 & 1.58 & 1.54 & 1.49 \\
\hline DN3/TN3 & 1.68 & 1.45 & 1.70 & 1.50 & 1.75 & 1.40 \\
\hline
\end{tabular}

Table 4: Calculated swelling parameters. $D N$ and TN in each dataset share the same $J_{s 1}^{1}$ and $J_{s 1}^{2}$ values while $J_{s 2}^{1}$ and $J_{s 2}^{2}$ values are only applicable to TN samples. Note that $J_{s 2}^{3}=1$ for all networks. $\phi_{2}^{1}$ and $\phi_{2}^{2}$ are volume fractions in DN samples, whereas $\phi_{3}^{1}, \phi_{3}^{2}$ and $\phi_{3}^{3}$ are for TN samples.

\begin{tabular}{|c|c|c|c|c|c|c|c|c|c|}
\hline Sample & $J_{s 1}^{1}$ & $J_{s 2}^{1}$ & $J_{s 1}^{2}$ & $J_{s 2}^{2}$ & $\phi_{2}^{1}$ & $\phi_{2}^{2}$ & $b_{3}^{1}$ & $\phi_{3}^{2}$ & $\phi_{3}^{3}$ \\
\hline DN1/TN1 & 2.41 & 9.94 & 0.64 & 2.66 & & 0.58 & 0.10 & 0.14 & 0.76 \\
\hline DN2/TN2 & 3.65 & 12.17 & 0.76 & 2.55 & & 0.73 & 0.08 & 0.23 & 0.70 \\
\hline DN3/TN3 & 5.36 & 14.71 & 1.00 & 2. & .19 & 0.81 & 0.07 & 0.29 & 0.64 \\
\hline
\end{tabular}

When no photophores are incorporated into the polymer network there is no light emission data from which the chain length distribution can be extracted. However, since these MNEs use the same density of crosslinker in the filler network (EAe1.45) the expected distribution will be similar to that of the network with photophores. Using the same form of the distribution (Eq. (34)), the values of $N_{\min }=6.2, a_{f}=0.6$, and $b_{f}=0.18$ are determined based on generating the best fit to the stressstretch data for all DN and TN elastomers (6 independent samples), which only differ slightly from the values extracted from Figure 4.

The results of the fitting are shown for the three datasets in Figure 9, Figure 10, and Figure 11 respectively. Each figure shows a comparison between predicted and measured stress-stretch relations for DN and TN elastomers. The model is in reasonable agreement with the experimental data in all 
cases. A direct comparison between the stresses and damage predicted by our model for the three datasets is presented in Figure 12, which illustrates the effect of prestretch on the mechanical response. In Figure 12a) the stresses are presented for three DN elastomers. DN3, which has the largest prestrech (Table 2), exhibits an upturn in stress at the smallest stretch. As the prestretch is decreased, the stress upturn occurs at larger stretches. Similar conclusions can be drawn from the TN data in Figure 12b). A comparison between the damage evolutions for the three TNs is shown in Figure 12c), where damage begins to occur at lower stretches for MNE with larger prestretches. 
a)
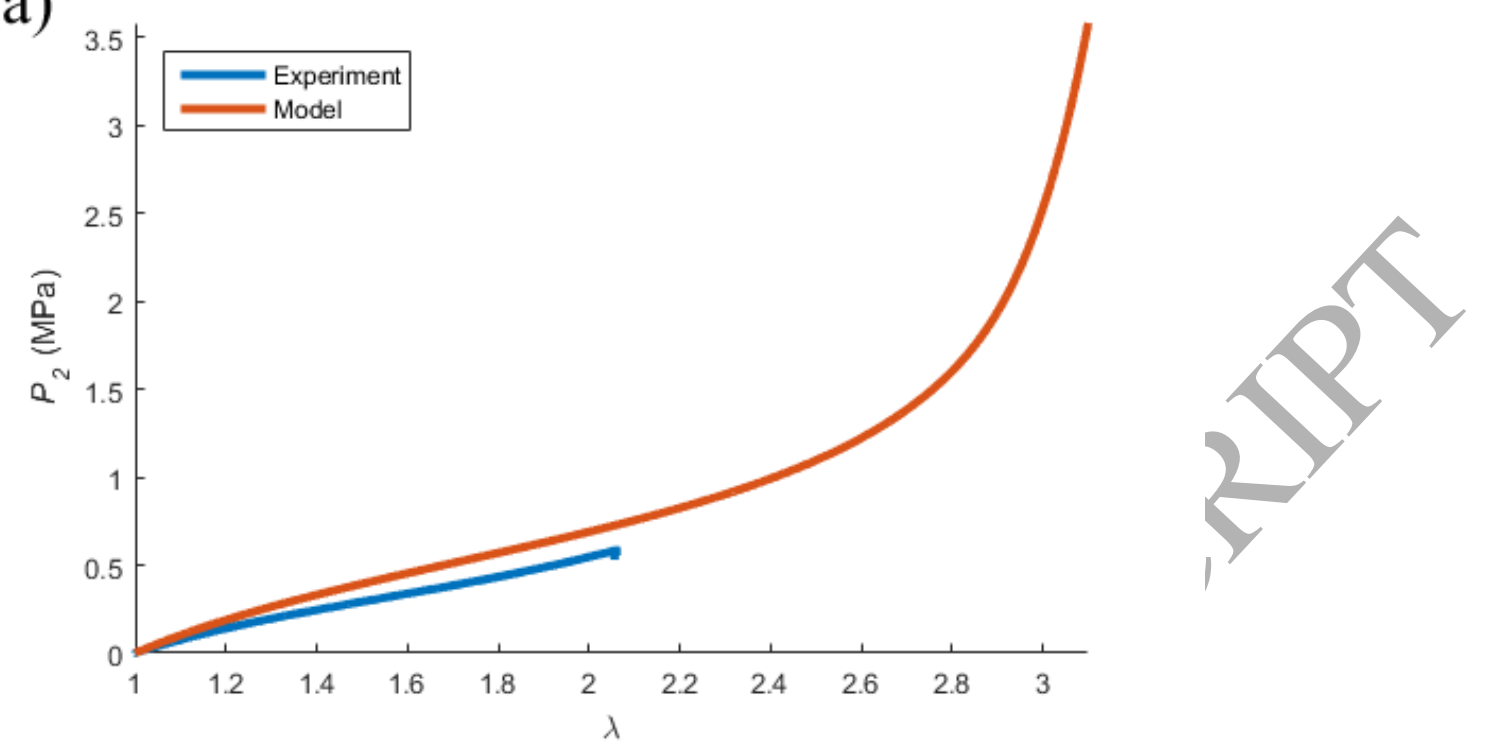

b)

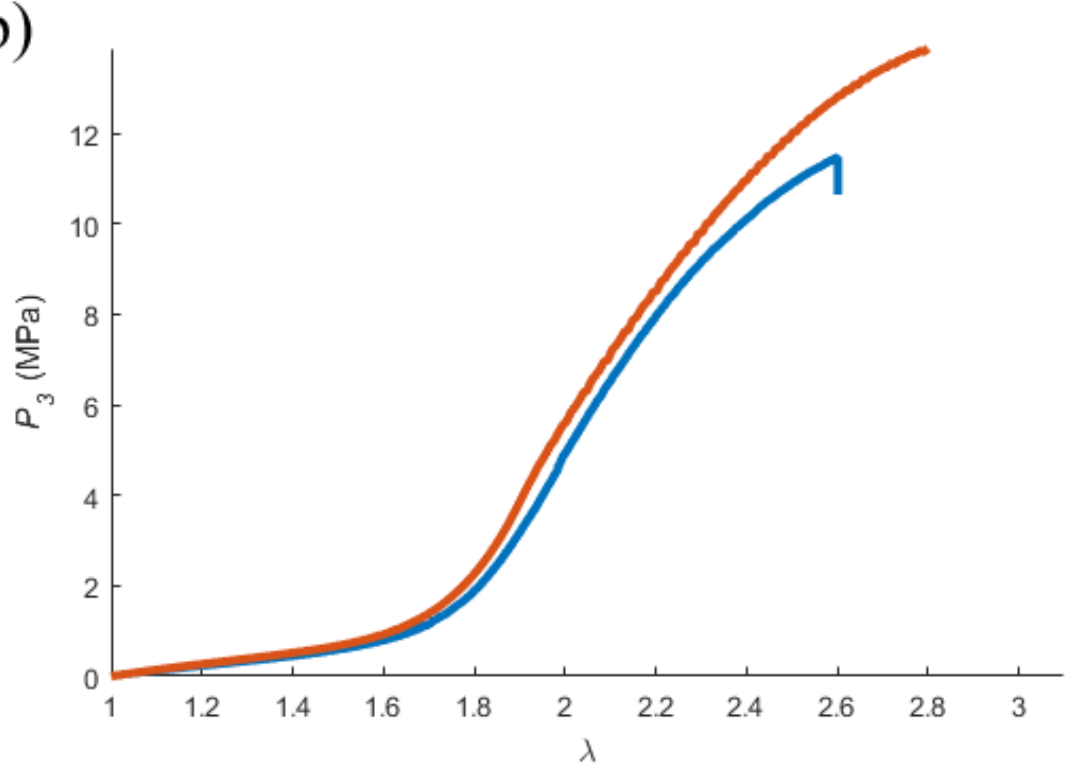

Figure 9 Engineering stress plotted against stretch for uniaxial extension of (a) DN elastomer (b) TN elastomer. Experimental data (from dataset DN1/TN1) and model fit are shown. 
a)
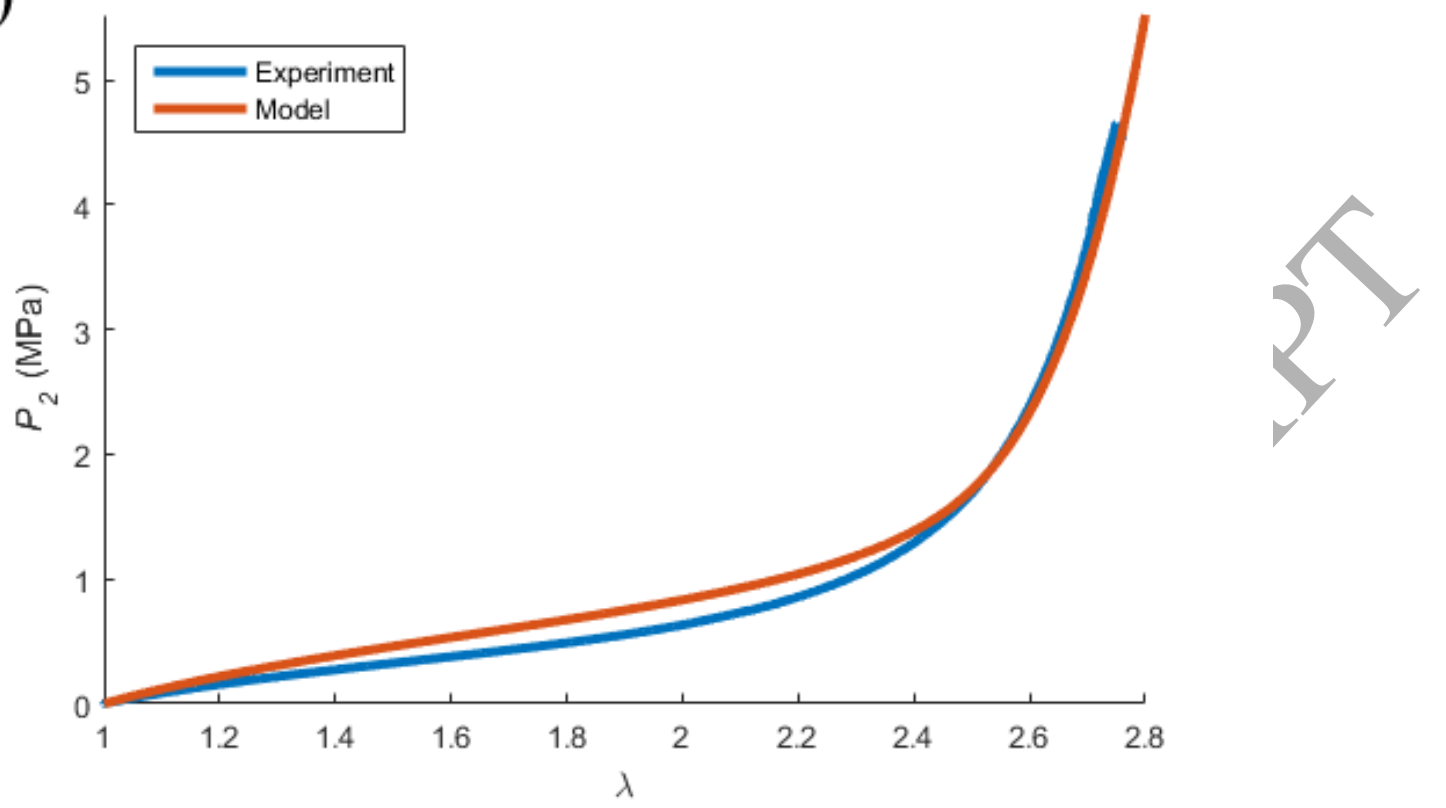

b)

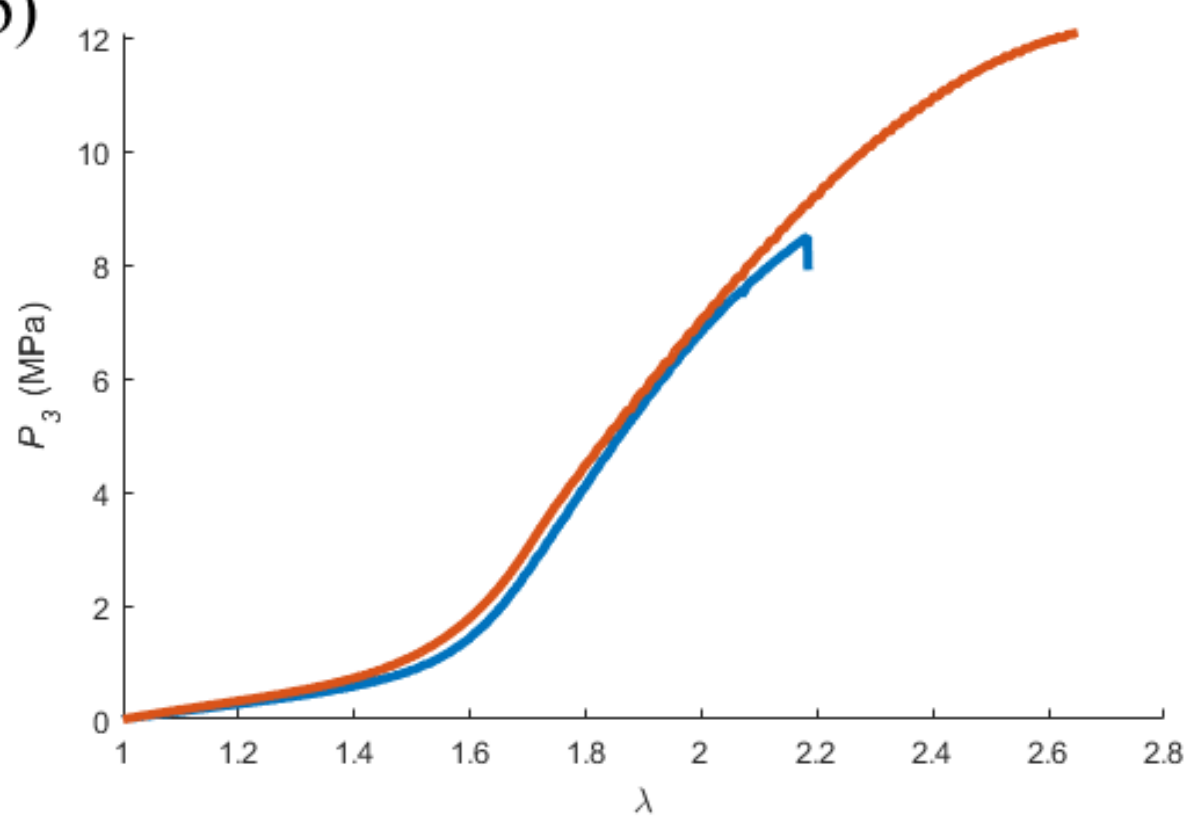

Figure 10 Engineering stress plotted against stretch for uniaxial extension of (a) DN elastomer (b) TN elastomer. Experimental data (from the dataset DN2/TN2) and model fit are shown. 
a)

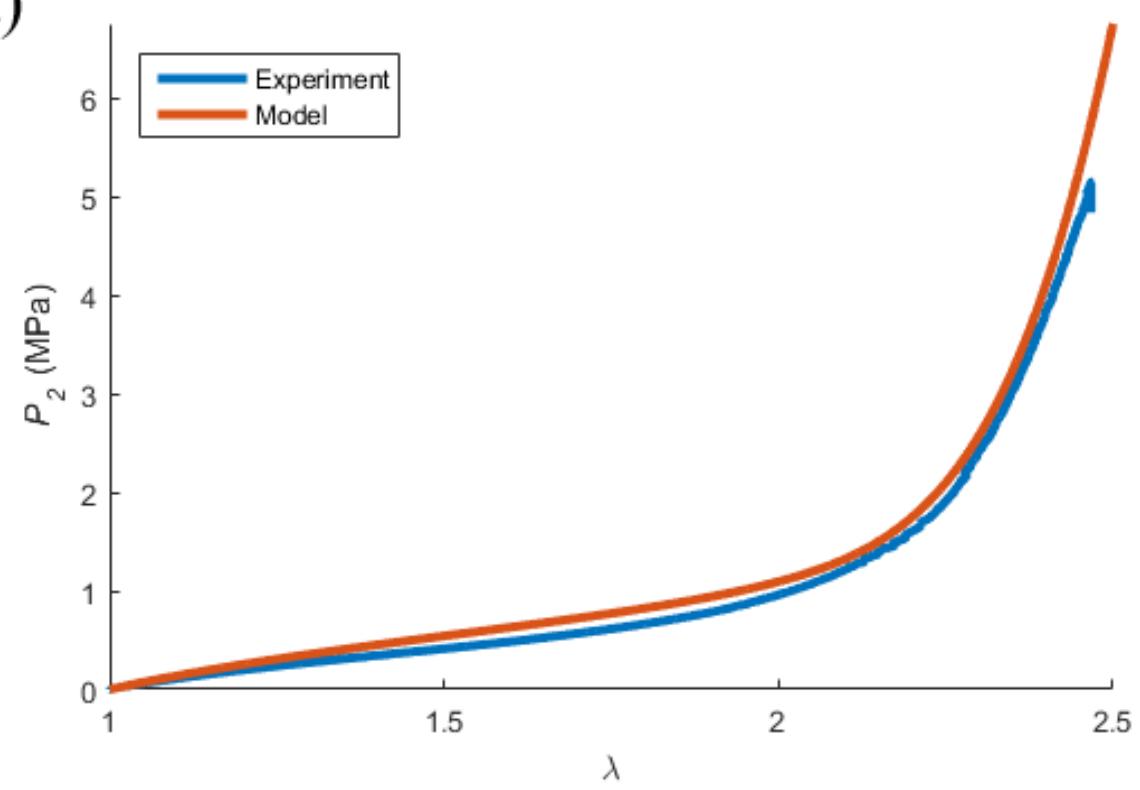

b)

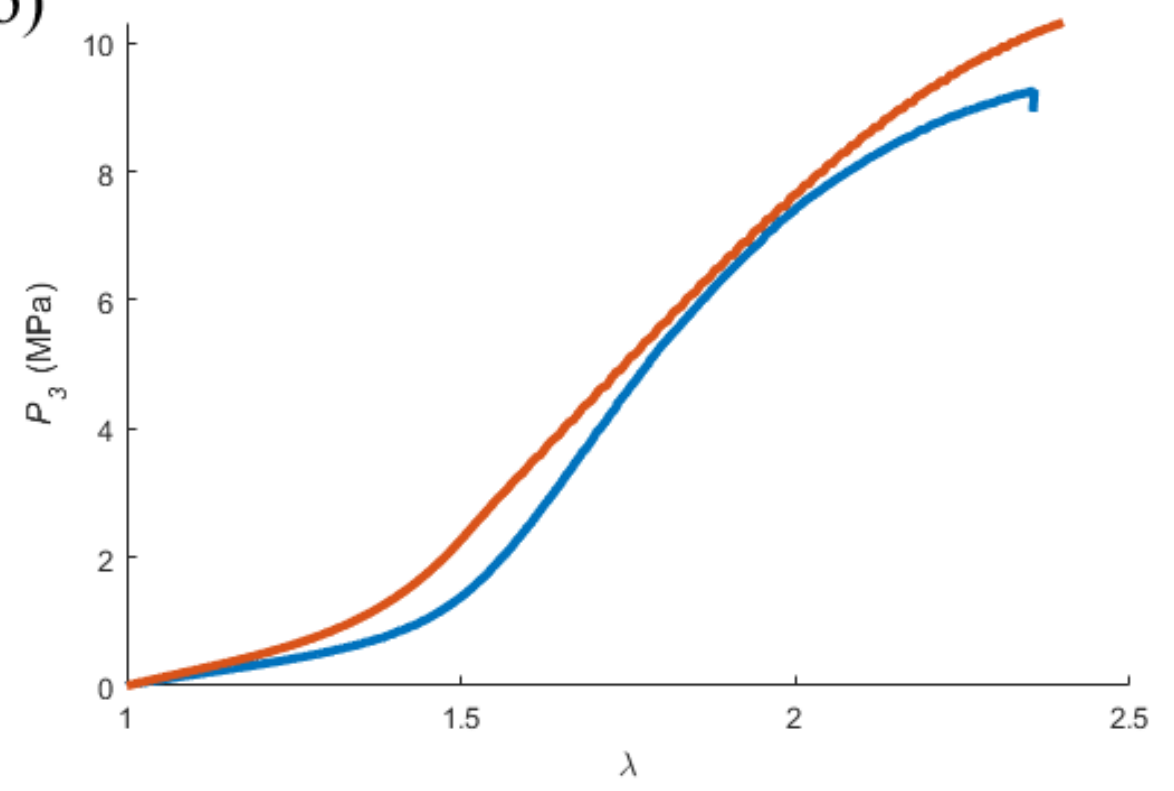


Figure 11 Engineering stress plotted against stretch for uniaxial extension of (a) DN elastomer (b) TN elastomer. Experimental data (from dataset dataset DN3/TN3) and model fit are shown.

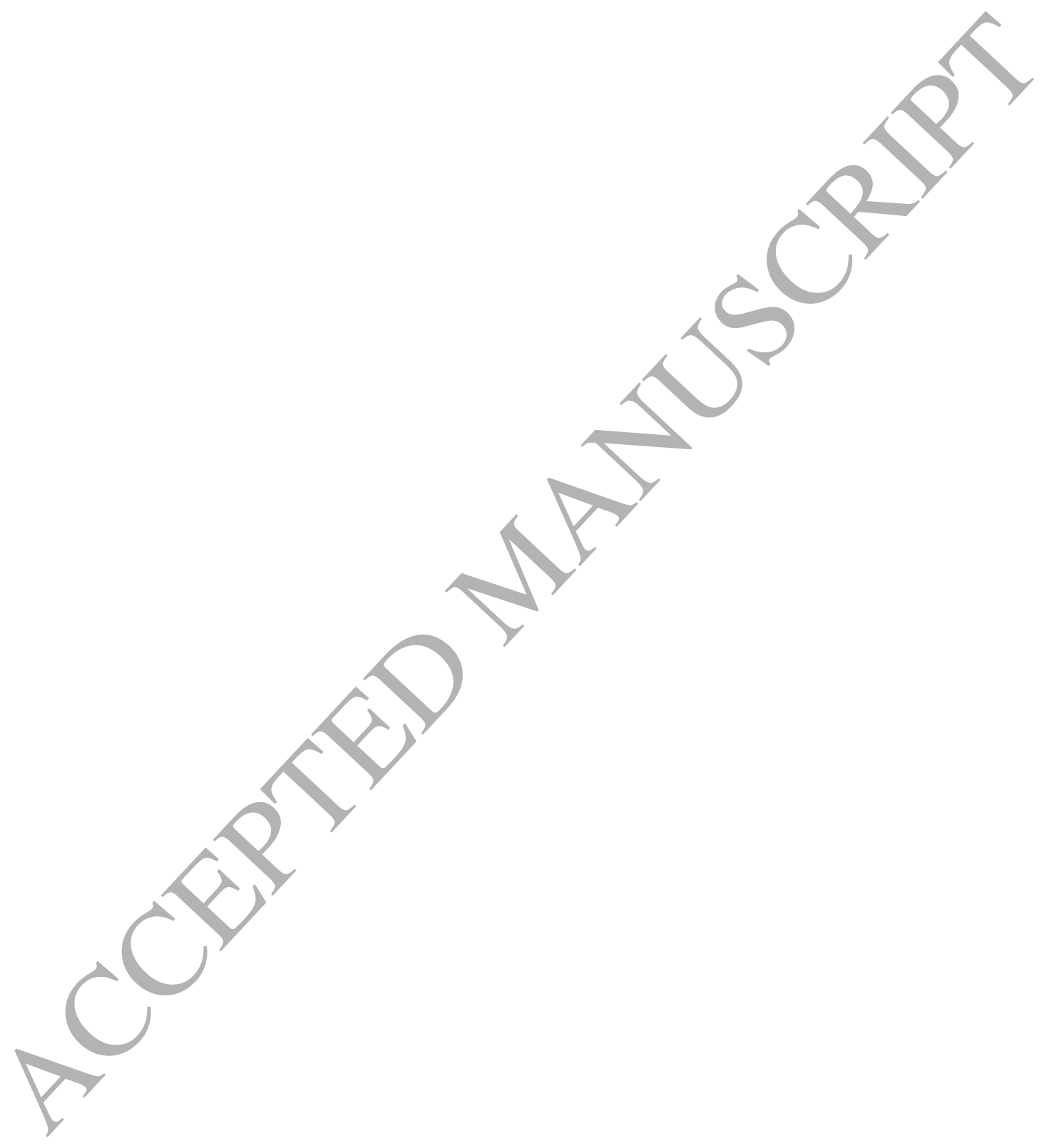


a)

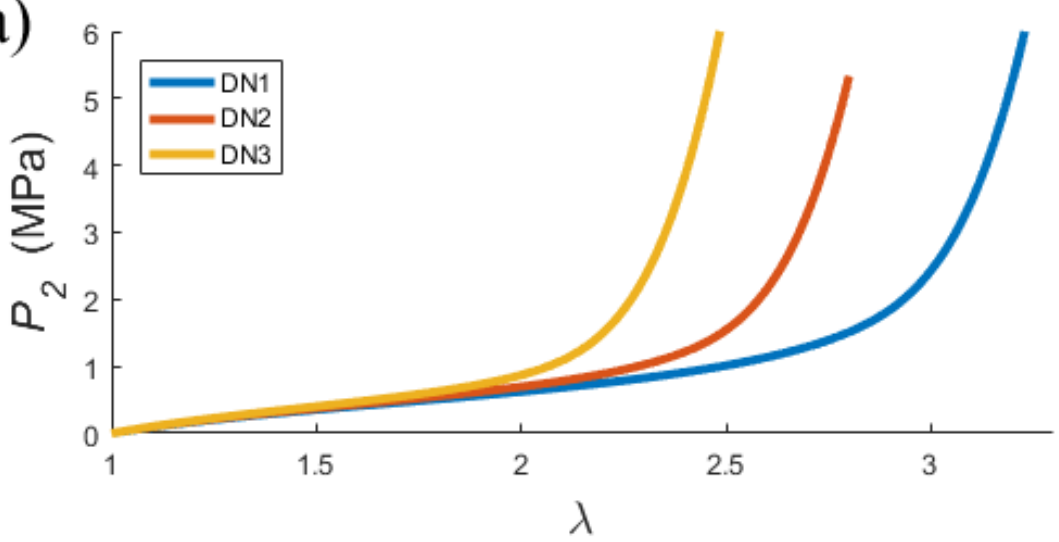

b)

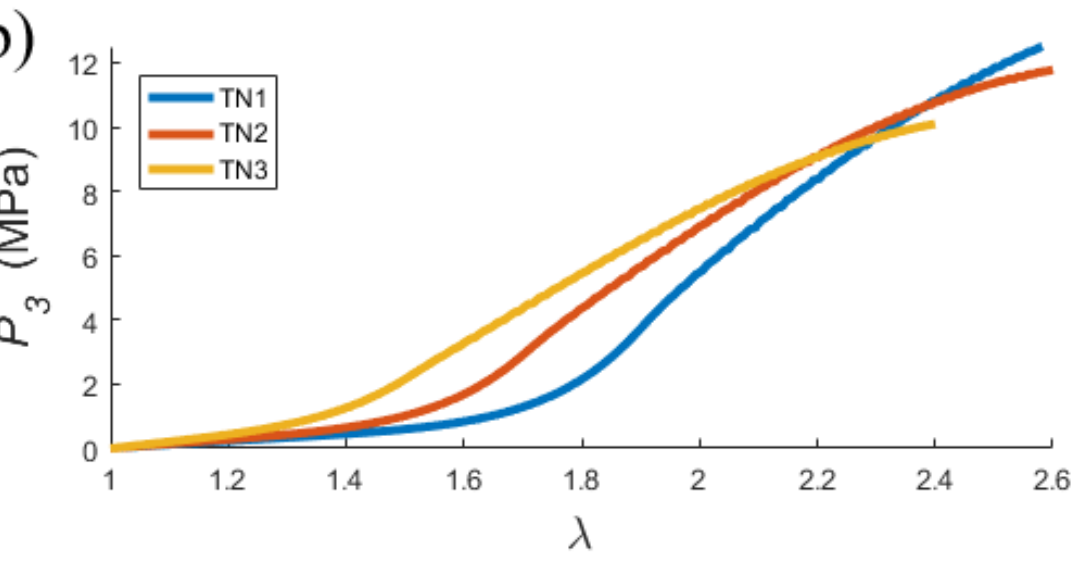

c)

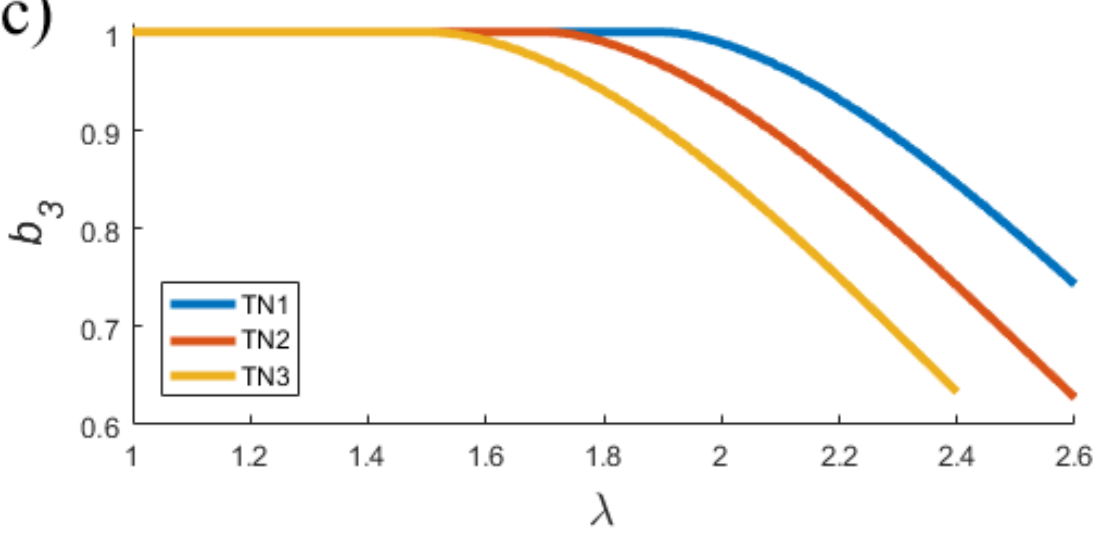

Figure 12: Model predictions for (a) engineering stress vs. stretch for DN elastomers; (b) engineering stress vs. stretch for TN elastomers; and (c) the evolution of damage (surviving chain fraction $b_{3}$ vs. stretch) for TN elastomers; all under uniaxial extension. Each subfigure contains three curves corresponding to the three different datasets. 


\subsection{Cyclic Loading of MNE}

The experimental data presented in Ducrot et al [13] for the MNE with photophores was for cyclic loading. It is interesting to apply the model under cyclic uniaxial extension to see how the unloading curves compare with the experimental data; studying cyclic loading can also further elucidate how the evolution of damage impacts the mechanical response of the MNE.

Consider the constant rate cyclic loading history shown in Figure 13a) where the amplitude of loading is increased after each cycle. In the experiments, three identical cycles were first performed before the amplitude was increased [13]; however, no noticeable change occurred in the $2^{\text {nd }}$ and $3^{\text {rd }}$ cycles so they have been omitted here to simplify the presentation of the numerical results. Engineering stress is plotted against stretch in Figure 13b), where loading and unloading curves are shown for both model prediction and experimental data of TNP. Similarly, the evolution of the damage variable is shown in Figure 13c). It is important to note that the loading envelope in the experiment (blue symbols) correspond to those in Figure 5b) and all the fitting parameters in the model remain unchanged from those used to obtain the fit in Figure 5b). No additional fitting was performed for the unloading branches.

To understand these results, consider the path $\mathrm{A}-\mathrm{O}_{1}-\mathrm{A}-\mathrm{B}-\mathrm{O}_{2}$ in Figure 13c). Suppose the material has been loaded to reach the stretch at A for the first time. In reaching point A some damage has occurred as can be seen in Figure 13c) where $b_{3}=0.72<1$ at point A. When the material is then unloaded from $A$ to $\mathrm{O}_{1}$ it follows the lower "damaged" unloading path (red, $\mathrm{A}-\mathrm{O}$ ) in Figure 13b) rather than the higher "undamaged" loading path (blue). From A to $\mathrm{O}_{1}$ in Figure $13 \mathrm{c}$ ) we follow a horizontal curve because the damage variable depends on the maximum stretch in the history of the deformation (in this case the stretch at point A) instead of the current stretch. During the subsequent reloading $\mathrm{O}_{1^{-}}$ 
$A-B$, from $\mathrm{O}_{1}-\mathrm{A}$ we retrace the same path as during unloading since the stretch has not exceeded its previous maximum value (at point $A$ ) so no additional damage occurs. From A-B stretching the sample further establishes a new maximum stretch, and thus the damage evolves as seen in Figure 13c) where the damage variable decreases from 0.72 at point $A$ to 0.49 at point $B$. This damage results in a decrease in the slope of the stress-stretch curve in Figure 13b) at the transition (point A) from reload to additional stretch. Similarly, when we unload after reaching point B we follow the lower unloading path (red, B-O) and again the damage variable remains constant during unload. 

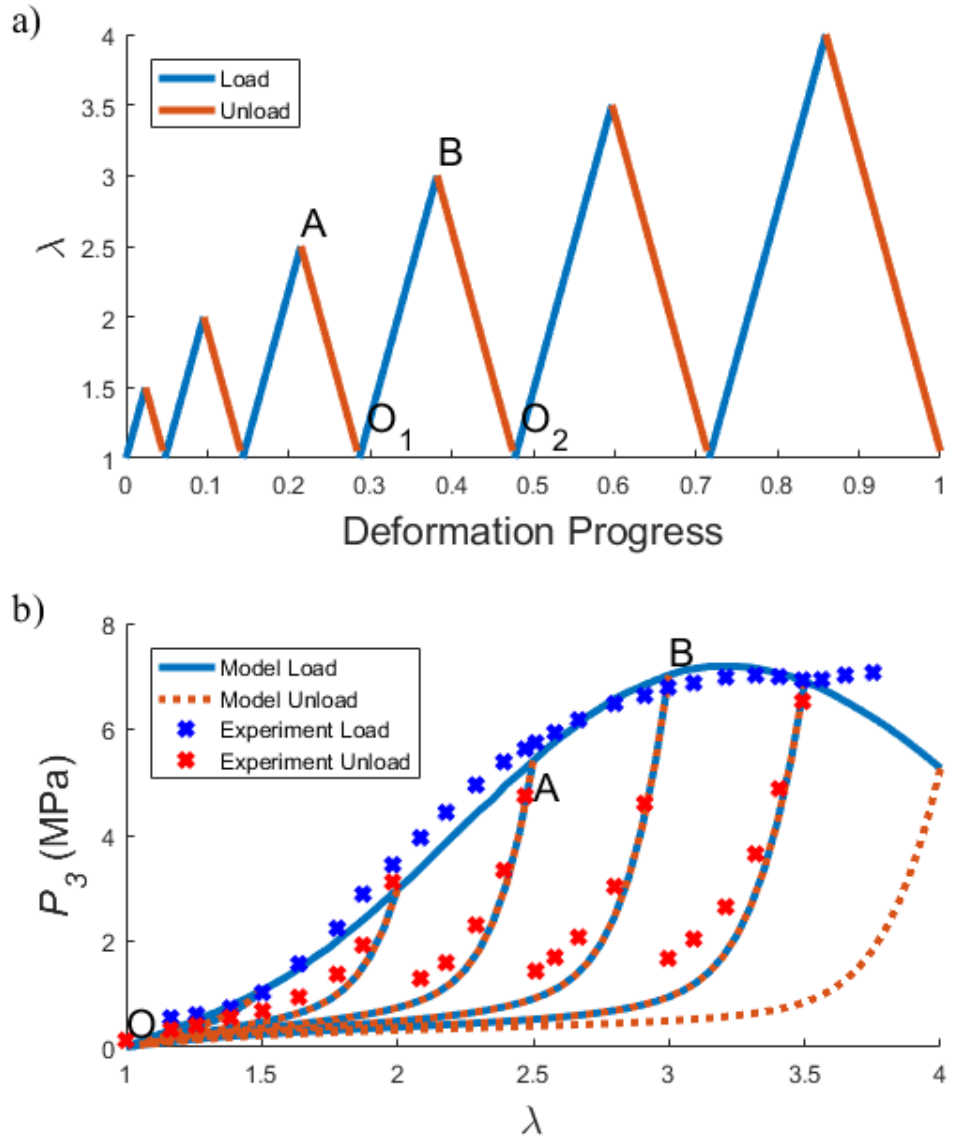

c)

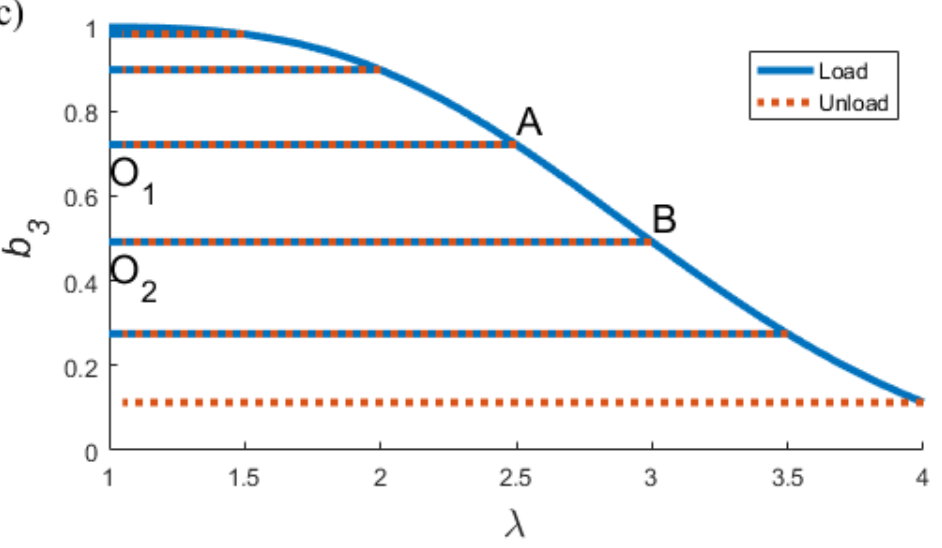

Figure 13: (a) Stretch plotted against deformation progress for constant rate cyclic loading where the amplitude is increased after each cycle. (b) Engineering stress plotted against stretch for cyclic uniaxial extension of TN elastomer. All parameters in 
the model are identical to those presented in section 4.3.1. Experimental data from Ducrot et al [13]. (c) The evolution of damage is shown by plotting the surviving chain fraction $\left(b_{3}\right)$ against stretch.

The cycle O-A-B-O in Figure 13b), included in the path we previously described, forms a hysteresis loop, and the area enclosed in this loop, $D_{\text {int }}^{c y c}=\oint_{c y c l e} P_{3} d \lambda$, has the physical interpretation of energy dissipation. The hysteresis during cyclic uniaxial extension was found to correlate, to some extent, with the size of damage zone in fracture experiments [13], and hence is an important quantity to examine in attempt to increase the fracture toughness of the material. In Ducrot et al. [13] this hysteresis was compared with the cumulative light emitted, whereas in our work, an analogous quantity would be the change in damage variable during a cycle

$$
\left|\Delta b_{3}^{c y c}\right|=-\oint_{\text {cycle }} \int_{1}^{\infty} f\left(N_{K}\right) \dot{b}\left(r_{\max }^{*}, N_{K}\right) d N_{K} d t
$$

An expression for the rate of energy dissipation in our model was obtained in the Supporting Information Section S2, $\dot{D}_{\text {int }}=-\phi_{N}^{1} \mu \int_{1}^{\infty} f\left(N_{K}\right) \dot{b}\left(r_{\text {max }}^{*}, N_{K}\right) N_{K} E_{c h}^{*}\left(r^{*}\right) d N_{K}$, so $D_{\text {int }}^{c y c}$ can be determined by either integrating $\dot{D}_{\text {int }}$ over a cycle or by using

$$
\left.D_{\text {int }}^{c y c}=-\phi_{N}^{1} \mu\right) \oint_{\text {cycle } 1}^{\infty} f\left(N_{K}\right) \dot{b}\left(r_{\max }^{*}, N_{K}\right) N_{K} E_{c h}^{*}\left(r^{*}\right) d N_{K} d t=\oint_{c y c l e} P d \lambda
$$

The numerical results for $\left|\Delta b_{3}^{c y c}\right|$ and $D_{\text {int }}^{c y c}$ are presented in Figure 14, for cycles 2-5 from Figure 13. In Ducrot et al [13] the cumulative light was found to vary with the mechanical hysteresis by a power of 
0.75 , while in Figure 14 a power of 0.63 provides a good fit which is in reasonable agreement with experiments.

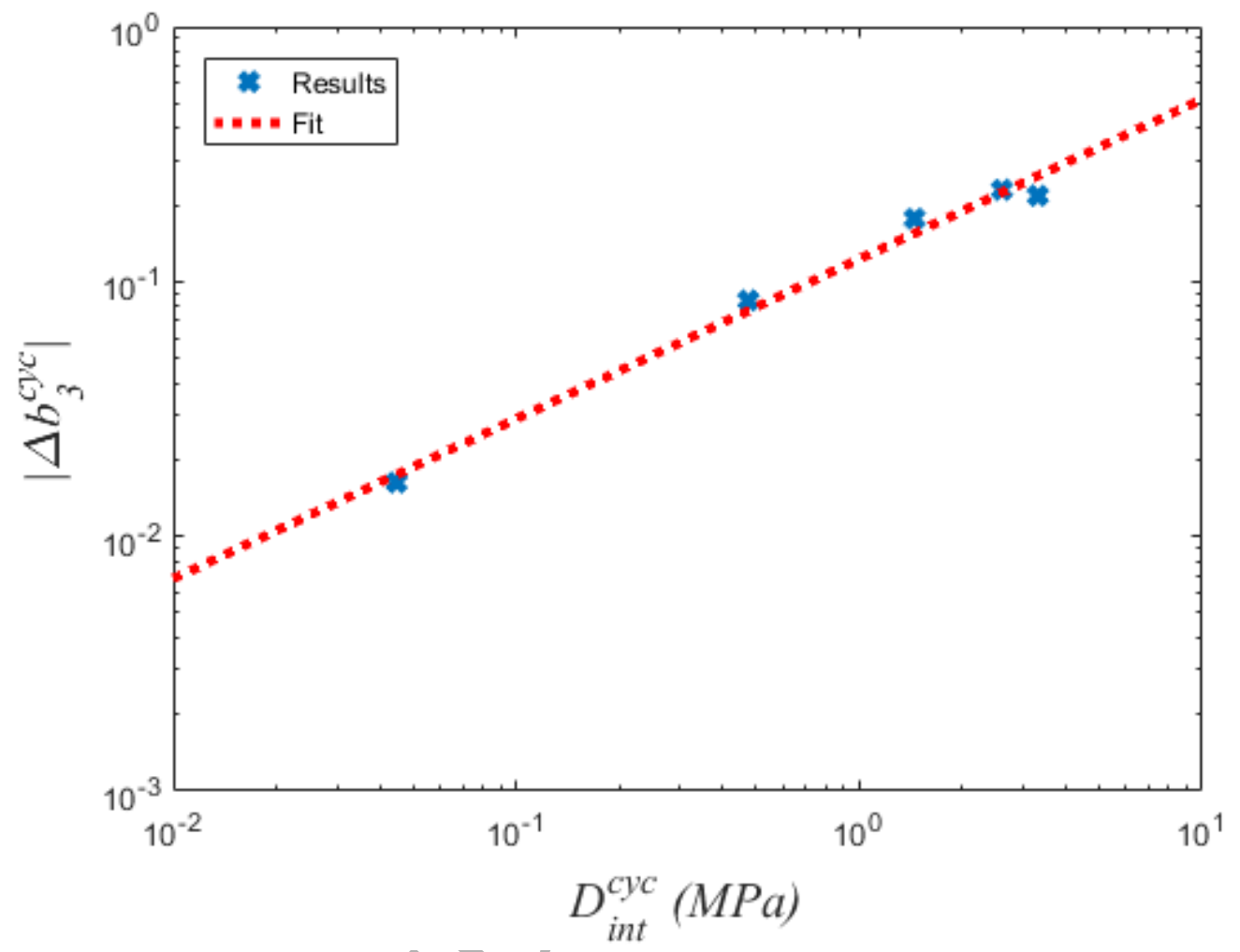

Figure 14: Decrease in damage variable per cycle plotted against energy dissipated per cycle. Symbols are from integration of numerical results obtained from the model. Dashed line is a linear fit to the model prediction, on the log-log scale.

\subsection{Further Discussion}

While our model predictions have shown good agreement with experiments, there are some discrepancies that warrant further discussion. First, in the uniaxial extension results in Figure 9, Figure 10, and Figure 11 for the TN elastomer, the model seems to overestimate the stress when the sample is about to break. A possible explanation for this is the potential material inhomogeneity not considered 
in the model. When we apply the model to an idealized uniaxial extension, the deformation is assumed to be perfectly homogenous, which is certainly not valid when the sample fails. Localized damage which grows near a pre-existing defect may also impact the overall stress of the specimen at the stretches leading up to failure. There is evidence to support this hypothesis in the TN light emission data [13]. Specifically, there is a peak in the light emission after which the intensity decreases with further stretching. In obtaining the chain length distribution from the light emission data (Figure 4), a distribution with a single peak was used, because it was assumed that a single damage mechanism occurs within the material. However, the experimental data also showed increased light emission intensity near failure which may indicate the possibility of additional damage mechanisms such as inhomogeneous deformation and localized damage around defects. This over-estimation does not exist in the DN data because the DN elastomers are expected to have lower fracture toughness, evidenced by the experiments of Ducrot et al. [13] where the damage zone in the area surrounding a crack tip [13] is much smaller in the DN elastomers than the TN elastomers. The lower fracture toughness could result in a more brittle failure once damage localizes at a defect; in this case, we do not anticipate a reduction in stress growth near the failure point.

Another discrepancy between the model and the experimental results lies in the unloading curves in Figure 13b), where the stress predicted by the model can be noticeably lower than measured stress. To elucidate this point, we compute the unstretched Young's modulus (see Supporting Information Section S2 for definition), i.e., the Young's modulus evaluated at $\lambda=1$. The ratio between the value at the beginning of cycle $i(i>1),\left(E_{o}^{N}\right)_{k}$, and that before the first cycle $\left(E_{o}^{N}\right)_{1}$ is evaluated and plotted in Figure 15 against the maximum stretch $\lambda_{\max }$ reached in that cycle. Here the loss of chains (Figure 13c)) translates to a reduction in Young's modulus. A plot similar to Figure 15 was presented in the Supporting Material of Ducrot et al [13], for a similar TN elastomer without photophores. In that 
work, after a maximum stretch of $\lambda_{\max }=2.4$ was reached in the cycle the unstretched Young's modulus decreased by approximately 20\%; from Figure 15 at $\lambda_{\max }=2.4$ the modulus decreases by approximately $50 \%$. This suggests the possibility that the model over predicts the rupture of chains. There are several potential explanations for the discrepancy. Firstly, one of the assumptions made in the model is that the contribution of each network to the stress is independent. While this may be a reasonable first-order approximation in modeling these materials, due to chain entanglements and a small degree of transfer reactions (e.g., the $(n+1)^{\text {th }}$ network reacting chemically with the $n$ networks previously formed), it is likely that damage in the filler network will result in dissipation in the matrix networks and these additional dissipation mechanisms may have some impact on the Young's modulus. An evidence of this is the small amount of residual strain observed in the experimental data [13], where after damage the material did not completely recover its initial configuration. A second possibility could be the existence of critical pathways in the filler network along which the rupture of bonds can result in the relaxation of many chains in the filler network. In this case the damage would not be isotropic and homogenous as assumed in this model. In fact, stretching pre-deformed samples perpendicular to the direction of initial deformation has yielded some light emissions [33] indicating that damage is not entirely isotropic. A third and related possibility is the coupling between chains in the filler network. There is no direct experimental evidence for this, but in an existing model [34] the rupture of chains was considered to effectively remove crosslinks and increase the length of existing chains in the material. Finally, the specific chain force relationship (Eq. (21)) is used for both loading and unloading. During loading the stress is dominated by chains with forces in the bond stretching regime. However, during unloading this is no longer true, and the force on the polymer chain can depend on its bending flexibility [29] which was not considered in Eq. (21). 


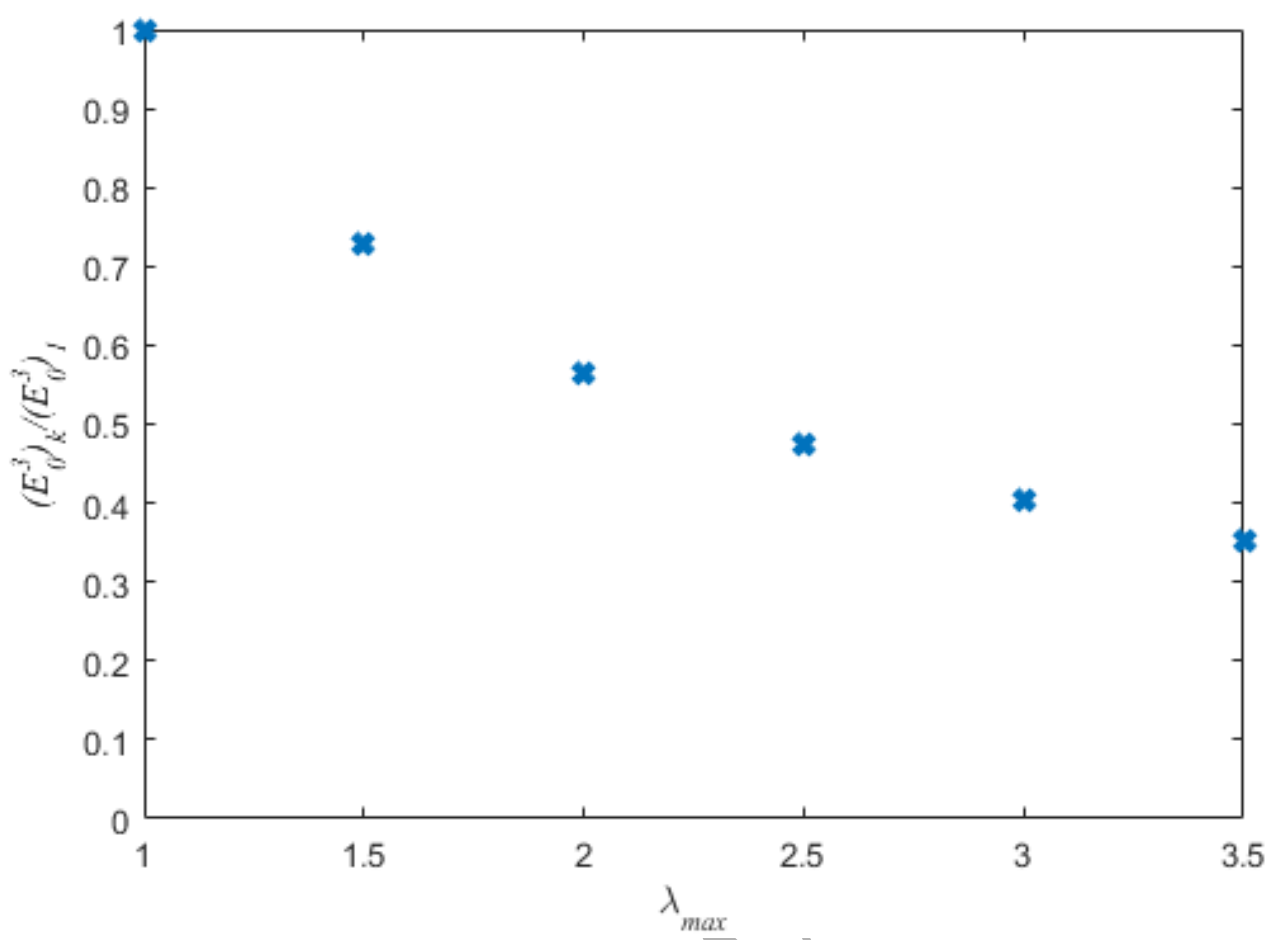

Figure 15: Evolution of unstretched Young's modulus at the beginning of cycle $k,(k>1)$ normalized by the unstretched modulus of a fresh sample, as a function of maximum elongation previously achieved by the sample.

Finally, we estimate the force and energy required to cause the rupture of a chain from the cyclic loading data in Figure 13. When a bond breaks we define the average force [35], $\left\langle F_{c h}\right\rangle=k_{B} T \int_{0}^{1} F_{c h}^{*}(r) d b / A_{o}$, and the average chain energy $\left\langle E_{c h}\right\rangle=k_{B} T \int_{0}^{1} N_{K} E_{c h}^{*}\left(r^{*}\right) d b$. For the data in Figure 13b) the following values are obtained: $\left\langle F_{c h}\right\rangle \sim 1.95 \mathrm{nN}$ and $\left\langle E_{c h}\right\rangle=1000-2000 \mathrm{~kJ} / \mathrm{mol}$ depending on the chain length. The force value is comparable to single chain pulling experiments for polymer chains [36], while the energy requires more discussion. The dissociation energy of the photophore bond was reported to be $150 \mathrm{~kJ} / \mathrm{mol}$ [13], which seems to be one order of magnitude smaller than the $\left\langle E_{c h}\right\rangle$ value obtained here. However, it is incorrect to make such a direct comparison, 
for several reasons explained below. Firstly, the bonds break via a transition state which is altered by the external force [33]. As a result, the energy that the bond will be excited to when it breaks can be significantly less than the dissociation energy. Secondly, when a bond on a polymer chain is ruptured the energy of the entire chain is dissipated [1] which includes the deformation of all the bonds. The shortest chains in the model had 6.5 Kuhn segments for PEA which corresponds to 78 bonds [31]. Using $\left\langle E_{c h}\right\rangle=1000 \mathrm{~kJ} / \mathrm{mol}$ and if all the bonds in a shortest chain are excited to approximately the same energy this implies $\sim 13 \mathrm{~kJ} / \mathrm{mol}$ for each bond, which is approximately $8.5 \%$ of the dissociation energy. Clough et al. [33] have found that typical C-C bonds store $15-18 \%$ of the dissociation energy before rupture, which is on the same order of magnitude as was estimated for the dioxetane bond in the photophore.

The model developed in this work provides a method to systematically study how prestretch impacts the mechanical response and damage evolution of a MNE. It is also instrumental to the design of MNE with optimized fracture toughness. Implementing the model into finite element simulations will allow us to explore a wide range of problems involving stress concentration and crack propagation, since this class of material can generate large damage zones [13] locally. It is therefore of interest to systematically investigate how the different prestretches will impact the size of the damage zone around cracks and the overall fracture toughness. In recent experiments it was observed that when a third swelling operation was performed to generate quadruple network elastomers, necking occurred in the samples during uniaxial extension [14]. The mechanism of this phenomenon is believed to be transfer of load from the filler network to the matrix network, which has only been observed to by stable when the matrix network volume fraction exceeds 25 times the filler network volume fraction [14]. To model this phenomena it will be necessary to extend the present model to consider stiffening and failure of the matrix networks. Furthermore, mechanoluminescence has been used to demonstrate that the rupture 
of chains does not occur homogenously within a sample during necking [14]; this behavior will be captured by finite element implementation of an extended version of this model in a future publication.

\section{Conclusion}

A continuum model is developed to capture the mechanical response of multinetwork elastomers synthesized by introducing matrix network(s) into an existing filler network. The swelling process prestretches the chains of the network formed in the previous step; such prestretch is incorporated into the model by basing the strain energy of each network on the combined deformation caused by swelling, drying and post-swelling deformation of the completed MNE. Because the chains in the heavily crosslinked filler network undergo the greatest amount of prestretch and often break during the deformation, separate material models are used for the filler and matrix networks. The filler network is modeled as a polydisperse network of breakable polymer chains with nonlinear chain elasticity, while the matrix networks are modeled using the generalized neo-Hookean model. With a few fitting parameters, this model provides a good match to the uniaxial extension, including cyclic loading, experimental data. Although the filler network only occupies a small volume fraction of the MNE, it contributes to the majority of the stress as confirmed by our model and recent experimental data [14]. It was found that a larger prestretch of the filler network causes the MNE to exhibit strain stiffening effect at a smaller stretch, and the damage due to chain rupture initiates at lower stretches. Finally, the hysteresis of thel stress-stretch curve during cyclic loading is found to correlate to the accumulation of damage during the cycle. 


\section{Acknowledgements.}

S.R.L and T.T. acknowledge financial support from the Natural Science and Engineering Research Council (NSERC), Canada Foundation for Innovation and Alberta Innovates Technology Futures. R.L. acknowledges the support from the $3 \mathrm{M}$ non-Tenured Faculty Award. P.M. and C.C. acknowledge financial support from DSM Ahead, Geleen, Netherlands.

\section{Nomenclature}

\begin{tabular}{|c|c|c|}
\hline $\mathrm{S}$ & Area of sample in plane of view & Dimension \\
\hline$A_{o}$ & Initial Kuhn length of polymer chain & {$[\mathrm{L}]$} \\
\hline$\alpha_{l}$ & Proportionality constant to relate light intensity to bond rupture & {$[\mathrm{M}][\mathrm{L}][\mathrm{T}]^{-2}$} \\
\hline$A_{f}$ & Parameter in chain length probability density function & - \\
\hline$a_{f}$ & Parameter in chain length probability density function & - \\
\hline$b_{f}$ & Parameter in chain length probability density function & - \\
\hline$b$ & Damage evolution function & - \\
\hline$b_{N}$ & Surviving chain fraction & - \\
\hline$\beta_{l}$ & Proportionality constant to relate light emission to damage evolution & {$[\mathrm{M}][\mathrm{L}][\mathrm{T}]^{-2}$} \\
\hline$\underline{\mathbf{B}}$ & Left Cauchy-Green deformation tensor for mechanical deformation of MNE & - \\
\hline$\underline{\mathbf{B}}_{N}^{i}$ & $\begin{array}{l}\text { Left Cauchy-Green Deformation tensor of multinetwork elastomer with } N \\
\text { networks, with respect to the relaxed configuration of network } i \text {. Takes into } \\
\text { account deformation by swelling, drying and mechanically deformation by } \\
\text { deformation gradient } \underline{\mathbf{F}} \text {. }\end{array}$ & - \\
\hline$\underline{\mathbf{e}}_{k}$ & Unit vector in $\mathrm{k}$ direction & - \\
\hline
\end{tabular}




\begin{tabular}{|c|c|c|}
\hline$E_{c h}^{*}$ & Nondimensional Helmholtz free Energy of stretched polymer chain & - \\
\hline$\xi_{j}^{m o n}$ & Volume fraction of monomers in swelling solution & - \\
\hline$F_{c h}$ & Tensile force acting on polymer chain & {$[\mathrm{M}][\mathrm{L}][\mathrm{T}]^{-2}$} \\
\hline$F_{c h}^{*}$ & Nondimensional Tensile force acting on polymer chain & - \\
\hline$\underline{\Phi}_{j}$ & Deformation gradient for $j$ th swelling and dying operation & \\
\hline$\underline{\Phi}_{j}^{\max }$ & Deformation gradient for $j$ th swelling to equilibrium operation & \\
\hline$\underline{\Phi}_{j}^{d r y}$ & Deformation gradient for $j$ th drying operation & - \\
\hline$\underline{\boldsymbol{\Phi}}_{j}^{i}$ & $\begin{array}{l}\text { Deformation gradient after } j \text { swelling and drying operations with respect to the } \\
\text { relaxed configuration of network } i \text {. }\end{array}$ & - \\
\hline $\mathbf{F}$ & Deformation gradient applied to completed MNE by mechanical loading. & - \\
\hline$\underline{\mathbf{F}}_{N}^{i}$ & $\begin{array}{l}\text { Deformation gradient of MNE with } N \text { networks, with respect to the relaxed } \\
\text { configuration of network } i \text {. Takes into account deformation by swelling, } \\
\text { drying and mechanically deformation by deformation gradient } \underline{\mathbf{F}} \text {. }\end{array}$ & - \\
\hline$f$ & Probability density function for chain length & - \\
\hline$\Delta F_{m}$ & Contribution of chain leng & - \\
\hline$g$ & Arbitrar & - \\
\hline$\underline{\mathbf{I}}$ & $2^{\text {nd }}$ order Identity tensor & - \\
\hline$I_{1}\left(\underline{\mathbf{B}}_{N}^{i}\right)$ & Firstinvariant of $\underline{\mathbf{B}}_{N}^{i}$ & - \\
\hline$I_{2}\left(\underline{\mathbf{B}}_{N}^{i}\right)$ & Second invariant of $\underline{\mathbf{B}}_{N}^{i}$ & - \\
\hline$I_{3}\left(\underline{\mathbf{B}}_{N}^{i}\right)$ & Third invariant of $\underline{\mathbf{B}}_{N}^{i}$ & - \\
\hline$i$ & Identifies network & - \\
\hline $\bar{j}$ & Identifies swelling operation & - \\
\hline
\end{tabular}




\begin{tabular}{|c|c|c|}
\hline$J_{s j}$ & Ratio of volume after to volume before $j$ th swelling and drying operation & - \\
\hline$J_{j}^{d r y}$ & Ratio of volume after to volume before $j$ th drying operation & - \\
\hline$J_{j}^{\max }$ & Ratio of volume after to volume before $j$ th swelling to equilibrium operation & - \\
\hline$J_{s j}^{i}$ & $\begin{array}{l}\text { ratio of the volume of the material after } j \text { swelling operations to the volume of } \\
\text { the material when the } i \text { th network was introduced }\end{array}$ & - \\
\hline$J_{N}^{i}$ & $\begin{array}{l}\text { ratio of the volume of the material when network } i \text { was introduced to its } \\
\text { current volume (with } N \text { networks) }\end{array}$ & \\
\hline$k_{B}$ & Boltzmann constant & {$[\mathrm{T}]^{-2}$} \\
\hline$\lambda$ & Stretch associated with uniaxial extension & - \\
\hline$\lambda_{s j}$ & $\begin{array}{l}\text { Isotropic stretch associated with } j \text { th swelling and drying operation (ratio of } \\
\text { thickness after to before } j \text { th swelling and drying operation) }\end{array}$ & - \\
\hline$\lambda_{j}^{d r y}$ & $\begin{array}{l}\text { Isotropic stretch associated with } j \text { th drying operation (ratio of thickness after } \\
\text { to before } j \text { th drying operation) }\end{array}$ & - \\
\hline$\lambda_{j}^{\max }$ & $\begin{array}{l}\text { Isotropic stretch associated with } j \text { th swelling operation (ratio of thickness after } \\
\text { to before } j \text { th swelling operation) }\end{array}$ & - \\
\hline$\lambda_{0}$ & Isotropic stretch of $1^{\text {st }}$ network due to swelling & - \\
\hline LI & Light emission intensity & {$[\mathrm{M}][\mathrm{L}][\mathrm{T}]^{-3}$} \\
\hline M & Num & - \\
\hline $\mathrm{m}$ & Identifies chain length bin & - \\
\hline$m_{i}$ & Mass of ith network & {$[\mathrm{M}]$} \\
\hline$m_{N}$ & Mass of multinetwork elastomer with $\mathrm{N}$ networks & {$[\mathrm{M}]$} \\
\hline$n_{M}$ & Parameter in constitutive model for matrix networks. & - \\
\hline$N$ & Number of networks & - \\
\hline$N_{K}$ & Number of Kuhn length is a polymer chain & - \\
\hline$N_{\min }$ & $\begin{array}{l}\text { Minimum number of Kuhn segments in chain length probability density } \\
\text { function }\end{array}$ & - \\
\hline
\end{tabular}




\begin{tabular}{|c|c|c|}
\hline $\bar{N}_{K, m}$ & Average number of Kuhn lengths per chain or bin $\mathrm{m}$ & - \\
\hline$N_{K, m}$ & number of Kuhn lengths per chain at start of bin $m$ & - \\
\hline$N_{K, m+1}$ & number of Kuhn lengths per chain at end of bin $m$ & - \\
\hline$p$ & Lagrange multiplier & {$[\mathrm{M}][\mathrm{L}]^{-1}[\mathrm{~T}]^{-2}$} \\
\hline$p^{i}$ & Lagrange multiplier for network $i$ & , \\
\hline$r$ & End to end distance of polymer chain & [L] \\
\hline$r^{*}$ & Fractional extension of polymer chain & \\
\hline$r_{\max }^{*}$ & $\begin{array}{l}\text { Maximum achieved fractional extension of a polymer chain during } \\
\text { deformation history }\end{array}$ & - \\
\hline$r_{p k}^{*}$ & Fractional extension where & - \\
\hline$P_{N}$ & $\begin{array}{l}\text { Nonzero engineering stress component for MNE with } N \text { networks, under } \\
\text { uniaxial extension }\end{array}$ & {$[\mathrm{M}][\mathrm{L}]^{-1}[\mathrm{~T}]^{-2}$} \\
\hline$P_{N}^{i}$ & $\begin{array}{l}\text { Nonzero engineering stress component for network i for MNE with } N \\
\text { networks, under uniaxial extension }\end{array}$ & {$[\mathrm{M}][\mathrm{L}]^{-1}[\mathrm{~T}]^{-2}$} \\
\hline$\psi^{i}$ & $\begin{array}{l}\text { Volume fraction that network } i \text { occupied in the material when it was first } \\
\text { introduced }\end{array}$ & - \\
\hline$\phi_{N}^{i}$ & in a multinetwork elastomer with $N$ networks & - \\
\hline$\underline{\boldsymbol{\sigma}}_{N}$ & Cauchy stress tensor for MNE with $N$ networks & {$[\mathrm{M}][\mathrm{L}]^{-1}[\mathrm{~T}]^{-2}$} \\
\hline$\underline{\boldsymbol{\sigma}}_{N}^{i}$ & Cauchy stress tensor for network $i$ in MNE with $N$ networks & {$[\mathrm{M}][\mathrm{L}]^{-1}[\mathrm{~T}]^{-2}$} \\
\hline$\sigma_{N}$ & $\begin{array}{l}\text { Nonzero true stress component for MNE with } N \text { networks, under uniaxial } \\
\text { extension }\end{array}$ & {$[\mathrm{M}][\mathrm{L}]^{-1}[\mathrm{~T}]^{-2}$} \\
\hline$\sigma_{N}^{i}$ & $\begin{array}{l}\text { Nonzero true stress component for network i for MNE with } N \text { networks, under } \\
\text { uniaxial extension }\end{array}$ & {$[\mathrm{M}][\mathrm{L}]^{-1}[\mathrm{~T}]^{-2}$} \\
\hline$D_{j}$ & Thickness of sample after $j$ - 1 swelling and drying operations & [L] \\
\hline$t$ & time & {$[\mathrm{T}]$} \\
\hline
\end{tabular}




\begin{tabular}{|c|c|c|}
\hline$\mu$ & $\begin{array}{l}\mu=v k_{B} T, \text { parameter in constitutive model for } 1^{\text {st }} \text { network. Related to } \\
\text { shear modulus. }\end{array}$ & {$[\mathrm{M}][\mathrm{L}]^{-1}[\mathrm{~T}]^{-2}$} \\
\hline$\mu_{M}$ & $\begin{array}{l}\text { Parameter in constitutive model for matrix networks. Related to shear } \\
\text { modulus }\end{array}$ & {$[\mathrm{M}][\mathrm{L}]^{-1}[\mathrm{~T}]^{-2}$} \\
\hline$U_{N}$ & Strain energy density of mutinetwork elastomer with $N$ networks & {$[\mathrm{M}][\mathrm{L}]^{-1}[\mathrm{~T}]^{-2}$} \\
\hline$U^{i}\left(\underline{\mathbf{B}}_{N}^{i}\right)$ & $\begin{array}{l}\text { Strain energy density for network } i \text { in a multinetwork elastomer with } N \\
\text { networks, if the network were to occupy the entire material when introduced. }\end{array}$ & \\
\hline$T$ & Absolute temperature & \\
\hline$v$ & $\begin{array}{l}\text { Volumetric density of load bearing chains in reference configuration o } \\
\text { material. }\end{array}$ & {$[\mathrm{L}]^{-3}$} \\
\hline$v_{m}$ & $\begin{array}{l}\text { Volumetric density of load bearing chains in reference configuration of } \\
\text { material in bin } \mathrm{m} \text {. }\end{array}$ & {$[\mathrm{L}]^{-3}$} \\
\hline$V_{j}$ & Volume of sample after $j$ - 1 swelling and drying operati & {$[\mathrm{L}]^{3}$} \\
\hline
\end{tabular}

\section{References}

(1) Lake, G.J.; Thomas, A.G. "The strength of highly elastic materials" Proc. R. Soc. Lond. A, 300 (1967) 108-119

(2) Creton, C.; Ciccotti, M. "Fracture and adhesion of soft materials: a review" Rep. Prog. Phys., 79 (2016) $57 \mathrm{pp}$

(3) Bhowmick, A. K. "Threshold fracture of elastomers. Journal of Macromolecular Science" Reviews in macromolecular Chemistry and Physics, C28 (1988) 339-370.

(4) Zhang, T., Lin, S., Yuk, H.; Zhao, "X., Predicting fracture energies and crack-tip fields of soft tough materials" Extreme Mech. Lett., 4 (2015) 1-8

(5) Mullins, L.; Tobin, N.R. "Theoretical Model for the Elastic Behavior of Filler-Reinforced Vulcanized Rubbers" Rubber Chemistry and Technology, 30 (1957) 555-571.

(6) Ogden, R.W., Roxburgh, D.G, "A pseudo-elastic model for the Mullins effect in filled rubber" Proc. $R$. Soc. Lond. A, 455 (1999) 2861-2877 
(7) Gent, A.N, "Adhesion and strength in viscoelastic solids. Is there a relationship between adhesion and bulk properties" Langmuir, 12 (1996) 4492-4496

(8) Gong, J.P.; Katsuyama, Y.; Kurokawa, T.; Osada, Y. "Double-Network Hydrogels with Extremely High Mechanical Strength" Adv. Mater., 15 (2003) 1155-1158

(9) Gong, J.P “Why are double-network hydrogels so tough?” Soft Matter., 6 (2010) 2583-2590

(10) Nakajima, T.; Kurokawa, T.; Furukawa, H.; YU, Q. M., Tanaka, Y; Osada, T; Gong, J.P “Super tough gels with a double network structure" Chinese Journal of Polymer Science., 27 (2009) 1-9

(11) Yang, C. Y.; Wang, M.X.; Haider, H.; Yang, J. H.; Sun, J.Y, Chen, Y. M.; Zhou, J.; Sou, Z. "Strengthening Alginate/Polyacrylamide Hydrogels using various multivalent cations" ACS Appl. Mater. Interfaces, 5 (2013) 10418-10422

(12) Li, J.; Illeperuma, W. R. K.; Sou, Z.; Vlassak, J.J. "Hybrid hydrogels with extremely high stiffness and toughness" ACS Macro. Lett., 3 (2014) 520-523

(13) Ducrot, E., Chen, Y., Butlers, M., Sijbesma, R.P., Creton, C. "Toughening Elastomers with Sacrificial Bonds and Watching them Break". Science, 344 (2014) 186-189

(14) Millereau, P., Ducrot, E., Clough, J.M., Wiseman, M.E., Brown, H.R., Sijbesma, R.P. and Creton, C., "Mechanics of elastomeric molecular composites." Proceedings of the National Academy of Sciences, (2018) p.201807750.

(15) Bacca, M.; Creton, C.; McMeeking, R.M. "A model for the Mullins effect in multinetwork elastomers", J. Appl. Mech., 84, (2017) 121009.

(16) Knowles, J.K, "The finite antiplane shear field near the tip of a crack for a class of incompressible elastic solids" Int. J. Frac., 13 (1977) 611-639

(17) Ducrot, E.; Creton, C. "Characterizing Large Strain Elasticity of Brittle Elastomeric Networks by Embedding Them in a Soft Extensible Matrix" Adv. Funct. Mater., 26 (2016) 2482-2492

(18) Millereau, P. "Large Strain and Fracture of Multiple Network Elastomers", These de Doctorat De L’Universite Pierre et Marie Curie. 22 May 2017

(19) Holzapfel, G.A., "Nonlinear Solid Mechanics: A Continuum Approach for Engineering” (2000) John Wiley and Sons: West Sussex

(20) Lavoie, S.R.; Long, R.; Tang, T. "A rate dependent damage model for elastomers at large strain", Extreme Mech. Lett., 8 (2016) 115:124 
(21) Nakajima, T.; Fukuda, Y.; Kurokawa, T.; Sakai, T.; Chung, U.-i.; Gong, J. P. "Synthesis and Fracture Process Analysis of Double Network Hydrogels with a Well-Defined First Network." ACS Macro Letters, 2 (2013) 518-521

(22) Silberstein, M.N.; Cremar, L.D.; Beiermann, B.A.; Kramer, S.B., Martinez, T.J., White, S.R., Sottos, N.R. "Modeling mechanophore activation within a viscous rubbery network" J. Mech. Phys. Solids. 63 (2014) 141-153

(23) Miehe, C.; Goktepe, S.; Lulei, F. "A micro-macro approach to rubber-like materials-Part I: the nonaffine micro-sphere model of rubber elasticity" JMPS 63 (2014) 141-153

(24) Arruda, E.M, Boyce, M.C. "A Three-Dimensional Constitutive Model For The Large Stretch Behavior of Rubber Elastic Materials." J. Mech. Phys. Solids., 41 (1993) 389-412

(25) Hong, et al., "A theory of coupled diffusion and large deformation in polymeric gels" J. Mech. Phys. Solids, 56 (2008) 1779-1793

(26) James, H. M.; Guth, E. "Statistical Mechanics of Rubber Elasticity" J. Chem. Phys., 21 (1953) 10391049

(27) Treloar, L.R.G, "The Physics of Rubber Elasticity" 3rd edition (1975), Clarendon Press, Oxford

(28) Ghatak, A.; Vorvolakos, K.; She, H.; Malotky, D.L.; Chaudhury, M.K, "Interfacial rate processes in Adhesion and Friction" J. Phys. Chem. B., 2000, 104, pp 4018-4030

(29) Dobrynin, A.V., Carrillo, J.M Y., Rubenstein, M., “Chains are more flexible under tension”, Macromolecules, 43 (2010) 9181-9190

(30) Mao, Y.; Talamini, B.; Anand, L. "Rupture of polymers by chain scission", Extreme Mechanics Letters, 13 (2017) 17-24.

(31) Lavoie, S.R.; PhDThesis. University of Alberta January 2018.

(32) Rubinstein, M.; Panyukov, S. "Elasticity of polymer networks." Macromolecules, 35 (2002) 66706886

(33) Clough, J. M., C. Creton, S. L. Craig and R. P. Sijbesma "Covalent Bond Scission in the Mullins Effect of a Filled Elastomer: Real-Time Visualization with Mechanoluminescence." Advanced Functional Materials 26 (2016) 9063-9074.

(34) Zhao, X. "A theory for large deformation and damage of interpenetrating polymer networks." J. Mech. Phys. Solids 60 (2012) 319-332. 
(35) Dudko, O.L.; Hummer, G.; Szabe, A. "Intrinsic Rates and Activation energies from Single Molecule Pulling Experiments." PRL 96 (2006) 108101

(36) Schwaderer, P.; Funk, E.; Achenbach, F.; Wies, J.; Brauchle, C.; Michaelis, J. "Single Molecule Measurement of the strength of a Siloxane Bond", Langmuir, 24 (2008) 1343-1349

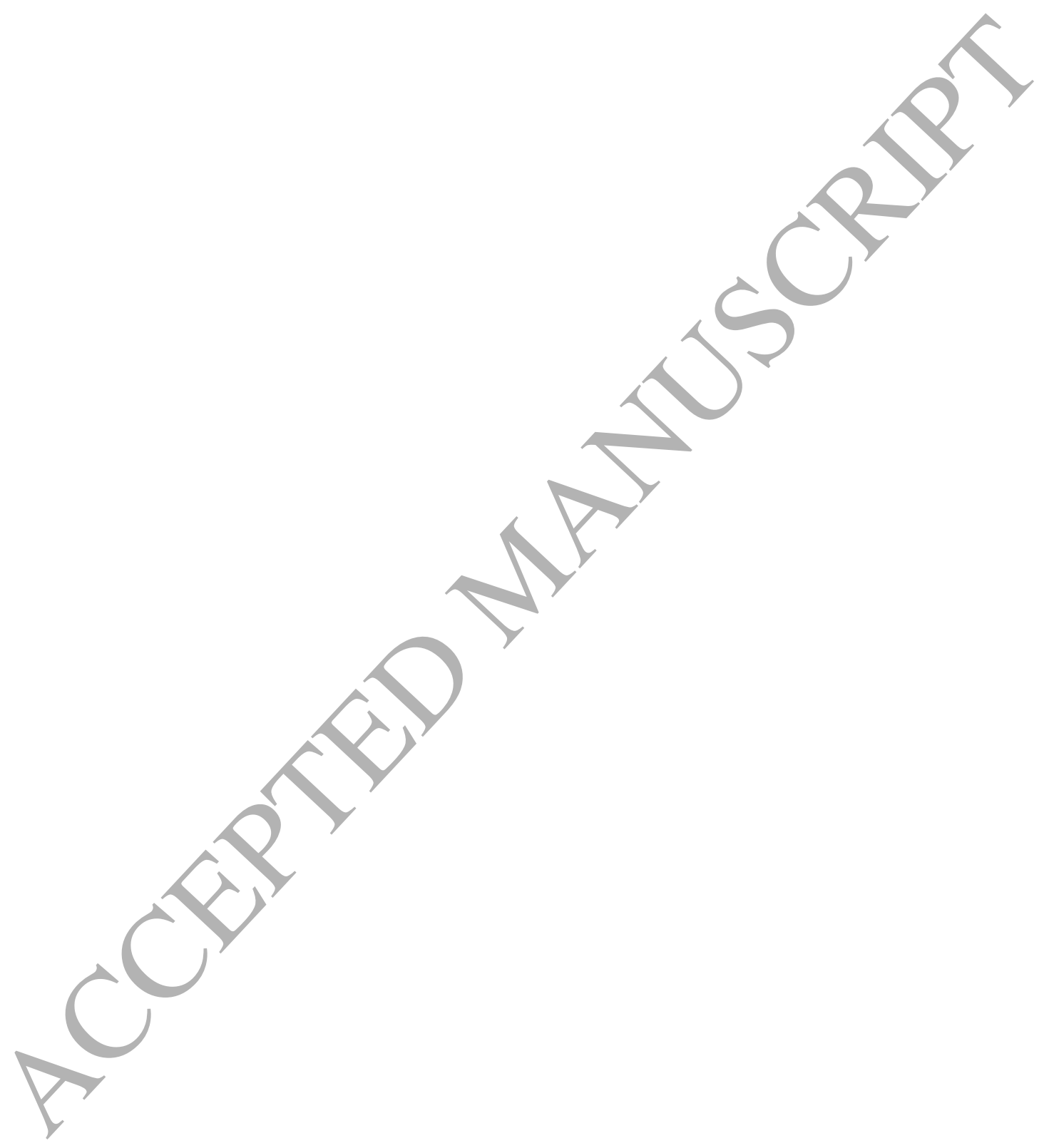

